\section{HSE}

Historia Social y de la Educación

Social and Education History

\section{Hipatia Press}

www.hipatiapress.com

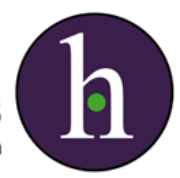

\title{
Volume 5, Number 3
}

\section{Hipatia Press}

www.hipatiapress.com

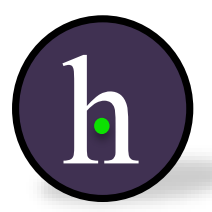

John Dewey: His Role in Public Scholarship to Educate for Peace- Charles F. Howlett \& Audrey Cohan

1939: The Reformist School of Catalonia goes to Exile- Salomó Marquès

Articles

Affirmative actions for minorities in India: Constrains and possibilitiesMohd Sanjeer Alam

Seeking new paradigms in aboriginal education research: methodological opportunities, challenges and aspirations- Marnee Shay

A philosophical examination of social justice and child poverty- Raquel Moreira

Culturally responsive leadership in Higher Education Promoting access, equity and improvement- Carlos Herrero 


\section{HSE}

Historia Social y de la Educación

Social and Education History

Instructions for authors, subscriptions and further details:

http://hse.hipatiapress.com

\section{John Dewey: His Role in Public Scholarship to Educate for Peace}

Charles F. Howlett ${ }^{1}$, Audrey Cohan ${ }^{1}$

1) Molloy College (USA).

Date of publication: October 23 2016

Edition period: October 2016 - February 2017

To cite this article: Howlett, C.F. \& Cohan, A. (2016). John Dewey: His role in public scholarship to educate for peace. Social and Education History 5(3), 203-222. doi:10.17583/hse.2016.2097

To link this article: http://dx.doi.org/10.17583/hse.2016.2097

\section{PLEASE SCROLL DOWN FOR ARTICLE}

The terms and conditions of use are related to the Open Journal System and to Creative Commons Attribution License (CC-BY). 


\section{John Dewey: His Role in Public Scholarship to Educate for Peace}

Charles F. Howlett

Molloy College (USA)
Audrey Cohan

Molloy College (USA)

\section{Abstract}

As 2016 is the centennial of Dewey's most famous work, Democracy and Education (1916), it is important to consider Dewey's role in public scholarship to educate for peace. Critical to an in-depth understanding of Dewey is recognition that the early twentieth century marked a transformational period in his views about war and peace. This paper addressed Dewey's less known political and social ideas during the rise of the "modern" American peace movement. In addition, Dewey's views of the role of education in a globalizing world are discussed. The research presented directly reflects global conflicts following World War I, while highlighting the disparity between war and peace.

Keywords: John Dewey, peace education, democracy 


\section{John Dewey: Su Papel en el Conocimiento Público para Educar para la Paz}

Charles F. Howlett

Molloy College (USA)
Audrey Cohan

Molloy College(USA)

\section{Abstract}

Ya que en 2016 se cumple el centenario del trabajo más reconocido de Dewey, Democracia y Educación (1916), es importante tener en cuenta el rol que Dewey jugó en la creación de un conocimiento público para educar para la paz. Para comprender en profundidad la obra de Dewey es esencial reconocer que los inicios del siglo XX supusieron un periodo que transformaría su visión sobre la guerra y la paz. Este artículo abordan las ideas políticas y sociales menos conocidas de Dewey durante el ascenso del movimiento "moderno" pacifista norteamericano. Además, se discute la visión de Dewey en relación al rol de la educación en un mundo globalizado. La investigación que se presenta refleja de forma directa los conflictos globales de después de la I Guerra Mundial mientas que se destaca la disparidad entre guerra y paz.

Keywords: John Dewey, educación para la paz, democracia 
s an educational theorist, John Dewey's work continues to be widely read and discussed by both pre-service and in-service teachers. However, critical to an in-depth understanding of

Dewey is recognition that the early twentieth century marked a transformational period in his views about war and peace. His less known social and political ideas-particularly his conception of democracy as a way of life-have generated debate and challenges from both the conservatives and liberals alike. This paper recounts Dewey's role in the peace movement and addressed his philosophies as related to global events.

The objectives of this paper are twofold: (a) to examine how closely Dewey's activities coincided with the rise of the "modern" American peace movement; and (b) to analyze Dewey's view on the role of education in a globalizing world. Both objectives entailed an examination of cultural resources for promoting both individual and communal growth. The research presented in this paper (Boydston, 1969-1991) directly reflects the "global conflict - fueled by political, territorial, ethnic, and ideological disputes which beckoned United States' engagement" (AERA, 2015, p.1) following World War I and highlights the interplay of research and social analysis.

In the aftermath of the Great War, Dewey became an ardent intellectual spokesperson on behalf of liberal internationalism and world peace. A strong supporter of President Wilson's progressive war aims, Dewey became disillusioned in the aftermath of the wrangling, which took place among the victorious Allies at the Treaty of Versailles. During the 1920s, he devoted his intellectual capital to the Outlawry of War movement resulting in the passage of the 1928 Kellogg-Briand Pact renouncing war as an instrument of national policy. Between the World Wars he also strongly supported the Committee on Militarism in Education, a peace group that opposed the creation of Reserve Officers Training Programs in colleges and universities across the United States. In addition, Dewey provided his own views as to how schools should promote the concept of internationalism and further cooperation among nation-states. Although not an "absolute" pacifist, Dewey's ideas and actions were based on his understanding of pacifism as a realistic and educative instrument necessary for battling the militaristic values and philistine patriotic views within society. Specifically, he did not consider bellicose values conducive to the democratic way of life. 


\section{Howlett \& Cohan-Dewey: His role in public scholarship}

In terms of our discussion, Dewey had the opportunity to address the role of schooling and peace during his journey to the Far East in 1919-1921 (Clopton \& Ou, 1973). In one particular lecture he delivered while in China, "The Cultural Heritage and Social Reconstruction," Dewey promoted three ground rules that were necessary if the schools were to create a feeling of democratic cooperation and world citizenship. The first rule and basic aim of education was for the school to create good citizens. When asked by the Chinese students to define what he meant by "good citizen," Dewey responded by listing four qualifications of the good citizen: (1) be a good neighbor and a good friend; (2) be able to contribute to others as to benefit from other's contributions; (3) be one who produced rather than one who merely shared in the production of others, from an economic standpoint; and lastly, (4) be a good consumer. According to Dewey's humanitarian and socially conscious outlook on life a "good citizen" was a person who contributed to the well-being of society. Above all, a "good citizen" was also one who appreciated the values of peaceful living by contributing to and sharing with his fellow citizens the fruits of society.

Dewey's second rule encouraged educators to create an atmosphere of harmony and friendliness whereby a feeling of world citizenship could be generated through the schools by making "students want to fulfill their duties to society, not from compulsion, but by curiosity and willingness, and out of love for their fellow men" (Clopton \& Ou, 1973, p. 211). But perhaps the most important rule was his last one, which incorporated the desire to acquaint students with the nature of social life and to the needs of society, as well as to their preparation for meeting these needs. A knowledge of one's environment and a willingness to eliminate its unworthy features, Dewey reasoned, was the main source of educational inspiration for the student. Social reconstruction, he believed, required more than sentiment. It demanded a general understanding of the nature of the problem and a willingness to adapt to new ways of thinking. Carter (2010) further noted that Dewey encouraged "this notion of purposeful engagement with conflicts in society" (p. 190) and he felt it should be part of the relevant educational curriculum. In each case, therefore, Dewey impressed upon his Chinese students a necessity for education to enhance the social, political, economic, and cultural institutions of a democratic society. "The school is the instrument," he concluded, "by which a new society can be built, and 
through which the unworthy features of the existing society can be modified" (Clopton \& Ou, 1973, p. 211).

Upon his return to Columbia University, New York, Dewey began calling for a new kind of curriculum, which would explore the theme of nationalism within an international context. What this curriculum should develop and encourage, he contended, is an attitude of world patriotism, not chauvinistic nationalism. "The teachers in our schools, and the communities behind the schools," he told his students at Teachers College and readers in the Journal of Social Forces, "have a greater responsibility with reference to this international phase of social consciousness and ideals than we have realized. As we need a program and a platform for teaching genuine patriotism and a real sense of the public interests of our own community so clearly we need a program of international friendship, amity and good will" (Dewey, 1923, p. 516). He insisted unhesitatingly,

We need a curriculum in history, literature and geography, which will make the different racial elements in this country aware of what each has contributed and will create a mental attitude towards other people which will make it more difficult for the flames of hatred and suspicion to sweep over this country in the future, which indeed will make this impossible, because when children's minds are in the formative period we shall have fixed in them through the medium of the schools, feelings of respect and friendliness for the other nations and peoples of the world. (Dewey, 1923, p. 516)

Dewey's arguments further highlighted the disparity between the two ends: war and peace. In terms of war, education teaches people to accept selfish behavior, promote authoritarian methods of rule, ignore moralistic reasons for good behavior, encourage coercion in the name of patriotic conformity, and comply with patterns of structural violence. In contrast, education for peace fosters responsibility, openness, innovation, selfmotivation, cooperative behavior, and barrier-free opportunities to pursue individual interests for the common good.

Dewey's intent was not to intellectualize the subject. Establishing a peaceful world order would never be accomplished by simply providing information and developing intellectual virtues. What he suggested is that one of the most important responsibilities for schools is to foster moral self- 


\section{Howlett \& Cohan-Dewey: His role in public scholarship}

discipline and humanistic self-fulfillment. The lesson he, himself, learned from the war was how effective schools were in promoting a singular patriotism. The final grade, however, was a failure.

Teachers did not communicate to their students that the ultimate goal was not the rightness of America's involvement in the war but the establishment of a global community rejecting the resort to armed conflict. "From the standpoint of...education, a large portion of current material of instruction," Dewey wrote in 1922, "is simply aside from the mark." No wonder, he continued, "Our schools send out men meeting the exigencies of contemporary life clothed in the chain-armor of antiquity, and priding themselves on the awkwardness of their movements as evidences of deepwrought, time-tested convictions." Is it any wonder that pupils "are ripe to be gulled, or that their attitude is one which merely perpetuates existing confusion, ignorance, prejudice and credulity" (Ratner, 1929, pp. 779-780). Since the United States helped win the war it was now America's responsibility to tear away the clothing of "chain-armor antiquity" and proudly don the robes of lasting world peace.

In terms of analyzing the role of education in a globalizing world, moreover, Dewey's involvement in the 1920s Outlawry of War movement is quite illuminating. Here we see Dewey applying his philosophical and educational theories on behalf of the "modern" American peace movement. The attempt to find common ground between the supporters of the newlyestablished League of Nations and those seeking to renounce war through juridical means reached its apex in the 1920s. This effort would culminate in 1928 with the signing of the Kellogg-Briand Pact or, as it was popularly known in European circles, the Pact of Paris. The attempt to outlaw war highlighted the efforts of both conservative and radical peace activists in which there were those advocating a fairer, peaceable world perfecting existing political and social structures based upon a slow and deliberate change, and those urging a more transformative world. The more radical peace activists exhorted new social structures and a redesigned political order carried out by mobilizing mass public opinion to adopt more drastic measures in order to force quick change outside of the normal diplomatic channels. The resulting peace pact "became a cultural icon as much as a policy objective for the peace movement," (Limberg, 2014, p. 396) and one 
of its most noted supporters was Dewey. The Outlawry campaign became his major contribution to postwar liberal internationalist thought.

Earlier efforts to develop world order based on international law and arbitration had been undertaken prior to the outbreak of war in 1914. In fact, for a good part of the nineteenth century, arbitration was the primary goal of internationalists and leading peace societies in both the United States and Europe. The notion of arbitration had been approached from different angles, but all were focused on promoting an understanding in which nations would agree to submit their differences to an impartial arbiter prior to resorting to armed conflict (Patterson, 1976).

The Outlawry of War crusade was primarily a moralistic-legalistic approach to international diplomacy. Relying on the means of creating overwhelming public support for the program - an approach characteristic of the "modern" peace movement's grassroots composition - the Outlawry of War proponents had three objectives: (1) outlaw war as a legal method of settling international disputes; (2) establish a code of international law that all nations would adhere to; and (3) create a court of justice similar to the US Supreme Court, which would encourage each nation to surrender its own war criminals - no matter how influential - to an international tribunal. Specifically, the immediate aim was not to eliminate war but to delegitimize it as the court of last resort (Ferrell, 1953).

The driving force behind the Outlawry of War crusade was a rich Chicago lawyer by the name of Salmon O. Levinson. Levinson, a graduate of Yale Law School in 1888, had been a very successful corporation lawyer. Originally a staunch supporter of Wilson's plan for a League of Nations, Levinson had gradually become disillusioned by what took place at Versailles. His increasing disenchantment after 1919 led to his conclusion that the use of sanctions against a nation was the equivalent of war. He noted that it was ludicrous to attempt to outlaw and abrogate war while at the same time threatening it as a means of enforcement (Stoner, 1943).

In order to build intellectual support for his idea he enlisted Dewey's help. Given Dewey's penchant for engaging in public issues impacting upon the democratic way of life, Levinson believed that Dewey could apply his "method of intelligence" to the cause of world peace. Thus Dewey's version of the Outlawry idea entailed using the applied intelligence to build the requisite moral and political awareness, which would achieve the realization 


\section{Howlett \& Cohan-Dewey: His role in public scholarship}

(conceptually and in application) that the system of war is detrimental to the demands of any situation. This would require, Dewey believed, changing the existing thinking on war, which considered it a legitimate institution. A code of law and a court - as both a means and an end-coincided with his pragmatic approach to world politics. Thus, the means - the moral judgment to create a court - could be interconnected with the end - the political will to back the court's decisions. To Dewey's way of thinking, Outlawry was a constitutive act of public engagement designed to replace long held acceptance of the brutal struggle between one absolute authority with another. According to one Dewey biographer, Westbrook (1991), "For Dewey, outlawing war was both an end of and a means to the democratization of politics, not only in the United States but throughout the world" (p. 269).

His attraction to the crusade was based on a genuine desire to unite Levinson's $(1918,1918$ a) legalistic approach to peace with his own desire to redirect society's moral and ethical sentiments against war. He believed that "a re-organization of international relations would serve to harmonize the ethics of nations with those of individuals and thus help to civilize international life" (DeBenedetti, 1968, p. 4; DeBenedetti, 1980). Dewey was committed, moreover, to the belief that a community of enlightened members could actively participate in their own self-creation. Outlawry of War, as both a social instrumentality and diplomatic weapon, Dewey maintained, offered the public an opportunity to demonstrate its commitment to make world peace an actuality.

Thus, the basic theoretical premise as well as pragmatic argument undergirding Dewey's support for the Outlawry plan, rested upon his assumption that the means proposed to implement this new idea was an educated public opinion-cognizant of morality as justice formulated through standards of societal consciousness. This public understanding would then recognize the need for internationalism and cooperation among nations. Such cooperation would also function as the means for designing a treaty outlawing war when signed. Ultimately, the Code of Law and Supreme Court, when finally created, would become effective and enduring instruments of international peace. At no time did Dewey contemplate the "chimerical possibility" of successfully outlawing war by a mere juristic declaration or by legal excommunication. The function and effectiveness of 
a World Supreme Court, in Dewey's opinion, rested not upon enforcement of sanctions but upon developing educated moral and ethical judgments - the means - of humankind.

Clearly, Dewey considered the Outlawry movement as an extension of his democratic social psychology. For Outlawry to take hold only the right cultural conditions would have to be established in support of the kinds of behavior that integrate emotions, ideas, and desires disposed to peaceful coexistence - those educated moral judgments. Instead of perfecting the art of war, nations and their peoples need to perfect the art of peace. Outlawry can assist in establishing a proper image of the world as an interdependent whole directed by political decisions aided by reasoned psychological, economic, and sociological knowledge of the probable reactions of different political systems capable of waging war.

It was Dewey's primary intention to see to it that reason would take precedence over emotion and blind trust; Outlawry was just the first step in the legal battle against war. The objective of the program was to work on the minds and dispositions of the public. If more people were taught that war was a crime against humanity coercive measures to prevent its recurrence would no longer be needed. Understanding would replace fear, and agreement would replace distrust. Quite clearly, the problem was not what reprisals a nation must fear for considering acts of blatant aggression but the moral and ethical incompatibility in undertaking such a course. If the internationalism of the modern world, in both its economic and psychological, and its scientific and artistic aspects, was to be truly liberated and made articulate, Outlawry of War was the most realistic, indeed the only realistic, means for firmly establishing "an international mind to function effectively in the control of the world's practical affairs" (Dewey, 1923a, p. 9). Thus, it would appear that Dewey's identification, association, allegiance, and participation in the Outlawry of War crusade was in complete agreement with his pragmatic approach to international peace. Perhaps the late historian Curti (1967) put it best when he wrote:

If Dewey's dedicated devotion to this program seemed naively idealistic to some of his contemporaries as well as to historians, it was nevertheless an important testimony to his conviction that war might be eliminated if the world stopped thinking in terms of war and that an 


\section{Howlett \& Cohan-Dewey: His role in public scholarship}

unlimited national sovereignty contradicted both common sense and social and human needs. (p. 1117)

Dewey's philosophical investment in the Outlawry principle also demonstrates clearly how he attempted to connect his political and social thought to the larger goal of world peace. Philosophically, Dewey insisted that the key issue for any successful understanding to acceptance of Outlawry was to inquire why the pervasiveness of war as a legalized method was considered as an established political institution. He believed that the public was unaware of this fact and, as part of its knowledge base, accepted it as reality. Removing the theoretical obstacle to a full appreciation for the Outlawry concept required people to look beyond the political connection between war and law. It necessitated that individuals revise their long established thinking, so that legal methods be used against war rather than for it. His philosophy was directed at using the experiential, not knowledge, as the means to achieve an end to war.

Understanding Dewey's position (Martin, 2002) derived from his belief that there first needed to be a general recognition of the problem of war, which had been promoted by political concerns and defined as part of power politics. Based on past experience, alliances and military buildups indicate that power politics itself represents a series of steps to armed engagement; each aspect increases the chances of conflict between equally competing states. Once the process of inquiry created awareness to this fact then the second development in the mind would follow. It would be a solid body of belief and will that the rule of law against war would replace the longestablished political acceptance of the use of military force. It would mark an important step forward in resolving disputes between established countries that feel threatened by new ones on the rise. He considered the idea of Outlawry as a test to discover whether the will of the people would be for war or peace. It was not a matter of providing a solution to the problem, but a method for removing those theoretical obstacles, which prevented it from being addressed.

Quite simply, he argued in favor the method of intelligence for overcoming the theoretical road blocks, which proposed that knowledge is based on the public's experience of dealing with problems and improving the prospects of collective action as it pertains to human affairs. Dewey's application of democratic theory, as tied to the debate between diplomacy 
and Outlawry, rested upon the public's authority as the instrument for all judgments. Overly optimistic, he pinned his hopes in the method of intelligence so that the process of inquiry would lead to the realization that Outlawry in practice represented the culmination of public engagement and democratic deliberation.

Public engagement and democratic deliberation also involved his views regarding militarism in education. After World War 1 a concerted effort was undertaken by the United States Military to establish a Reserve Officers Program on college campuses. This attempt to inaugurate a new era in civilian-military relations - a result of the war psychology — culminated in the National Defense Act of 1920. The act itself was an ambitious plan for bolstering the nation's military, with the underlying aim of not getting caught off guard if the threat of war should once again become a reality. This initiative provided for the establishment of over three hundred ROTC units with about 125,000 students participating in the program on college campuses throughout the country. In addition to the creation of the ROTC program, another provision of the act provided for the construction of summer training camps for youths. The program, optimistically referred to as Universal Military Training, began to admit prospective recruits in 1922, offering a combination of military and civic instruction to ten thousand young men for a thirty-day period. The program was a two-year course in military subjects with weekly drill instruction. Upon graduation an individual trainee would automatically receive a commission in the US Army. Furthermore, efforts were also undertaken to establish a voluntary training program for public high school students during after school hours. Its purpose was to establish a bridge for promoting citizenship awareness for the children of the millions of new immigrants arriving in America (Ekirch, 1956).

A primary reason why Dewey opposed the militarization of schooling was his firm belief that discipline was unsuited to classroom purposes. He opposed the authoritarian practices of military training as well as the emphasis on rigid conformity to rote drills. Such techniques, he believed, were monotonous and boring. To Dewey, education should be a creative and self-developmental process; any form of strict discipline ran counter to his views on progressive education, and rigid uniformity was unacceptable. A sense of libertarian values plus a belief in a self-developmental form of 


\section{Howlett \& Cohan-Dewey: His role in public scholarship}

education oriented to a moral way of thinking accounted for Dewey's consistent opposition to militarism in education. In fact, one of the more distinctive features of his book How We Think (first published in 1910) was the emphasis he placed on the importance of moral thinking as an essential character trait - certainly in response to the world situation facing future generations of students. "They are not the only attitudes that are important [open-mindedness, whole-heartedness, responsibility] in order that the habit of thinking in a reflective way may be developed," he wrote. "But the other attitudes that might be set forth are also traits of character, attitudes that, in the proper sense of the word, are moral, since they are traits of personal character that have to be cultivated." In other words, thinking should not be a mechanical process but rather a matter of "how we should live our lives as moral agents if we are to think effectively" (Dewey, 1971, p. 53).

Clearly, the mechanical, non-cognitive aspects of military training were in direct opposition to Dewey's pedagogy. Dewey had long criticized existing practices in education for placing too much emphasis on the mere symbols of knowledge and for being reluctant to make sufficient use of positive, firsthand engagement with experience. He noted that direct contact with experiences fostering cooperation, not conformity, should be the basis for learning and understanding. Teaching the art of the martial spirit, he argued, elevates the mere symbols of knowledge related to that subjectswords, uniforms, guns, and glory - and reinforces them in a philistine way.

His views on the subject date as far back as the 1890s when he established the Laboratory School at the University of Chicago just when the apparent effects of urbanization on education were surfacing. The introduction of military subjects and forms of physical education reminded him of the detrimental effects of a mass urban system of public schooling, which required rigid structure to accommodate the influx of immigrant children. Dewey believed that militarization in education creates a mechanized and bureaucratic system of learning, a system that will lead only to unquestioned obedience to the state and those in positions of authority. Dewey feared that without the presence of freedom in this setting, students' abilities to think critically would be compromised.

In a 1916 New Republic article titled "The Schools and Social Preparedness," prior to American military intervention in the war, he took direct aim at the preparedness advocates by questioning their sincerity and 
underscoring the additional burden such a program would place on teachers and pupils. "A few years from now our state legislatures may be besieged by ardent advocates of international peace," he wrote, "who will guarantee the future amity of the world if all children can have a fourteen weeks' course of lessons in 'peace."' Certainly, in light of the war, Dewey carefully considered the role that high school students might play, but he could not accommodate the idea that schools would be integral to the war effort: "Just now, however, the clamorers for preparedness have the speaker's eye, and two or three hours a week of drill exercise is to be made compulsory in high schools. ... All of this mechanical confidence in the mechanics of school programs is an ironic tribute to our national faith in the efficacy of education. Meantime it is hard on the schools." The results of such legislation will be "overburdened schools with...distracted teachers and pupils" standing "a good chance of being offered up a sacrifice on the altar of 'act first and think afterwards"' (Ratner, 1929, pp. 474-475).

Dewey championed the view that "[w]e are a pacific people and in the main a kindly disposed one; we regret the loss of life, the flames of hatred in Europe," although this would be transformed a year later with American military intervention in World War I. Dewey was slowly preparing the groundwork for future peace education endeavors, which he would undertake in the war's aftermath: "Unless the methods of critical discrimination which they [universities] foster extend into our secondary schools and thence, indirectly at least, into the elementary schools, we shall find democratic control tied to a course of inert drift alternating with periods of excited explosion. To make our schools the home of serious thought on social difficulties and conflicts is the real question of academic freedom, in comparison with which the topic which we have hitherto dealt with under that head is indeed academic." Fostering social change and freedom to act independently is the academic mission of schooling: "A nation habituated to think in terms of problems and of the struggle to remedy them before it is actually in the grip of forces which create the problems, would have an equipment for public life such as has not characterized any people." There is absolutely nothing wrong, he insisted, with connecting "this intellectual habit with coherent thinking in matters of foreign relations" (Ratner, 1929, pp. 474-475).

His consistent belief that militarism in schools perverts the positive 


\section{Howlett \& Cohan-Dewey: His role in public scholarship}

aspects of nationalism as a unifier of different cultures and understanding led to his direct involvement in the Committee on Militarism in Education, established in 1925. As a pubic intellectual Dewey worked with the committee in its efforts to accomplish two specific objectives: to act as a lobbying group seeking legislation to prohibit federal funds for compulsory military training courses for ROTC units on campus and to function as an educational propaganda agency. "We are now faced," he wrote prior to the committee's establishment, "by the difficulty of developing the good aspect of nationalism without its evil side; of developing a nationalism which is the friend and not the foe of internationalism." More importantly, he opined, "Since this is a matter of ideas, of emotions, of intellectual and moral disposition and outlook, it depends for its accomplishment upon educational agencies, not upon outward machinery. Among these educational agencies, the public school takes first rank" (Dewey, 1916/1980, p. 203).

Perhaps Dewey's most important educational contribution to the committee was lending his pen to composing introductory remarks to the organization's publications. For example, one of the final pamphlets published by the committee, Edwin C. Johnson's Mars in Civilian Disguise! (1939), Dewey wrote the foreword. Supporting Johnson's claim that the federal government's training program for student pilots is camouflage for "a definitely militaristic project," not a civilian one, Dewey launched into an unrestrained attack on the government's sincerity. "Public moneys," he charged, "are needed for the peaceful maintenance of an industrious and prosperous citizenship. But they are being diverted to the cause of war." By doing so, the methods employed by the federal government "strengthen the belief that the American opposition to war is being used by interested parties to sell the American people down the war-river. Under the name of defense, measures are proposed that have no sense unless the American people are being prepared to engage in war. Since the American people are opposed almost to a man to this idea, it is necessary to put blinders upon them in order to lead them toward war." Dewey cautioned readers to consider the response of institutions of higher learning as well: "Do they want the harnessing to be done under the claim that the measure is civilian? If persons in charge of colleges and universities favor this plan, what shall the American people with their strong opposition to being involved in war think about what the colleges and universities are doing?" (Dewey, 1939/1988, pp. 


\section{5-356).}

Dewey's contributions to post-World War I peace thought and to the subsequent development of peace education has largely been ignored by scholars. It should not be. Given the current state of world affairs, it is all the more stunning why those in the field of education have not taken a closer look at Dewey's intellectual contributions to peace thought and action. Regardless of his philosophical shortcomings in the political realm, belief in an altruistic human nature, and insistence that an educated public was capable of changing an entrenched political system, he nonetheless offered a different way of defining democracy: more fitting as an "instrument" of peace rather than making the world safe. He called for a civic engagement against war, noting that democracy is a disposition that seeks to bridge differences, form common interests, reflect critically on beliefs and values, and promote knowledge addressing the core challenges of a global village. As a public intellectual, Dewey separated himself from the application of disciplinary knowledge and expertise by insisting that passive communities become part of the public debate.

Dewey also helped make respectable the powerful secular impulse, which remodeled and reorganized the post-World War I peace movement. His call for peace between international states was tied to his seeking alternatives to institutionalized violence. He also aligned his thinking with radicalized nonpacifists who argued that war is less a social sin than it is a symptom of systemic social injustice. He legitimized the view that one does not have to be an absolute pacifist to be against war. He helped popularize the current feeling that one can still support their country but remain committed to promoting the idea of conflict resolution - not armed intervention-as the most desirable means for eradicating all forms of social oppression and disputes between nation states. In fact, since the Vietnam War this secularist attitude has grown and expanded to the point where, ideologically, many Americans subscribe to it.

Reevaluating his philosophy also made the transition easier for Dewey when he joined ranks with more liberal elements within the postwar peace movement. Reenergized and refocused, the "modern" peace movement witnessed a growing radicalization of pacifism; personal witness for peace became less inward and more outspoken in terms of social and political action. Liberal pacifism struck a responsive chord in Dewey's postwar 


\section{Howlett \& Cohan-Dewey: His role in public scholarship}

pragmatic approach to international politics and domestic reform. Traditional pacifists who had long asserted that the means determine the ends were in line with Dewey's philosophical position that ethical decisions, tied to nonviolent force, were now relative to the demands of time and place. Equally significant, the postwar peace reformers, condemning violence in contrast to the traditional dictates of nonresistance, were also willing to sanction some aspects of coercion as a means of redressing racial, social, and economic injustice.

As a matter of principle, the horrors associated with the destructiveness and massive casualties - combatant and civilian-of World War 1 had convinced him that war was an embodiment of collective behaviororchestrated and propagated by powerful interest groups who were able to influence the psychology of the masses in favor of armed conflictcorrupting the entire social order as well as its political structure. He thus began working from that principle to use his philosophy and educational theories to establish alternative means for resolving human conflicts and to develop forms of group harmony so that the means - the instrument of peace-might persist as an ongoing social dynamic in the lives of all individuals. In keeping with his understanding of the importance of community, moreover, he backed the concept of a global order for the larger society to emulate. And instrumentalism he viewed as a reform measure for replacing the political policies, social institutions, and cultural patterns that continued to prevent the triumph of lasting peace.

Antiwar activists, along with committed pacifists who now believed that peace required social reform as well as social order, tipped the scales in favor of Dewey's support. The modern (that is, postwar) peace activists were quick to point out that their predecessors represented an uncontroversial establishment reform effort. In their view American society and institutions would first have to fundamentally change if the United States wished to take a leading role in reforming international relations. More importantly, as historian Nigel Young (2013) observed, modern peace activists, not only added a moral dimension to their methods, but also "a theory of conflict and a dialectic of action in a struggle that became an 'experiment with truth': testing ideas through political dialogue, exemplary conduct, and communication during conflict, rather than through political violence. In the United States, Gandhi's ideas of nonviolent resistance blended with 
Reinhold Niebuhr's pacifism, John Dewey's pragmatism, and other strands of peace thought and civil disobedience" (Young, 2013, p. 160).

Furthermore, Dewey's rationale for supporting the "modern" peace movement as realistic, not utopian, is premised on his call for a democratized international system in which responsible policy makers would follow the lead of the public, managing peace through applied social justice and world agencies. The "modern" movement also rejected the "sentimental" nationalism of the prewar peace movement and the exclusivity of national self-determination in favor of a "higher" nationalism, which responded to the collective wisdom of the modern populace. Dewey certainly appreciated the "modern" peace movement's grassroots militancy and secularism as realistic alternatives to state-sponsored war; its increased acknowledgment of the economic causes of war; its willingness to challenge social elitist tendencies, within and without the movement; and its determination to initiate direct political, nonviolent action from the bottom up. The movement represented a desire to experiment with new ideas and tactics to accomplish its goals of world peace and social justice. Dewey's support was also tied to his realization that the United States could not escape the violence of modern war unless Americans were willing to assist in the reordering of international relations to mitigate national rivalries before they broke the chains of interdependence and drew the nation into another global conflict. He was indeed aware that public sentiment for building a lasting world peace was sincere and genuine, and he sought to cash in on it.

Instructively, it is incumbent upon readers to revisit Dewey's (1916) magnum opus, Democracy and Education, to capture the essence of his perception of national sovereignty, which he considered a major impediment to world peace. At the time he was writing this work he observed that,

Each [nation] is supposed to be the supreme judge of its own interests, and it is assumed as a matter of course that each has interests which are exclusively its own. To question this is to question the very idea of national sovereignty which is assumed to be basic to political practice.... (p. 97)

But that is exactly what he called upon educators to do for, 
This contradiction...between the wider sphere of associated and mutually helpful social life and the narrower sphere of exclusive and hence potentially hostile pursuits..., exacts of educational theory a clearer conception of the meaning of 'social' as a function and test of education than has yet been attained (Dewey, 1916, p. 98)

The message he left for future generations of educators for peace was for them to alter the environmental forces elevating the principle of national sovereignty as inviolable and replace it, as noted by him previously, with "...whatever binds people together in cooperative pursuits...apart from geographical limitations...[and the] provisional character of national sovereignty in respect to the...more fruitful association of intercourse of all human beings with one another must be instilled as a working disposition of the mind" (p. 98).

\section{Notes}

This article is based, in small part, upon a much larger study of Dewey's peace activism published by Southern Illinois University Press (2016) and is entitled, John Dewey, America's Peace-Minded Educator.

\section{References}

American Educational Research Association. (2015). 2016 Annual meeting call for submissions. Retrieved from http://www.aera.net/EventsMeetings/AnnualMeeting/2016AnnualMeetin gTheme/tabid/15861/Default.aspx

Boydston, J. A. (Ed.). (1969-1991). The collected works of John Dewey, 1882-1953. Carbondale, IL: Southern Illinois University Press.

Carter, C. C. (2010). Teacher preparation for peace education. In C. C.

Carter (Ed.), Conflict resolution and peace education (pp. 187-200). New York, NY: Palgrave Macmillan.

Clopton, R. W., \& Ou, T. C. (Eds.). (1973). John Dewey: Lectures in China, 1919-1920. Honolulu, HI: University of Hawaii Press.

Curti, M. (1967). John Dewey and nationalism. Orbis, 10, 1109-1117. Curti, M. (1968). The roots of American loyalty. New York, NY: Atheneum. 
DeBenedetti, C. (1968). American internationalism in the 1920's: Shotwell and the Outlawrists. (Unpublished doctoral dissertation). University of Illinois, Chicago: IL.

DeBenedetti, C. (1980). The peace reform in American history.

Bloomington, IN: Indiana University Press.

Dewey, J. (1916). Democracy and education. New York, NY: Macmillan.

Dewey, J. (1916/1980). Nationalizing education. In J. A. Boydston (Ed.),

The middle works of John Dewey, Vol. 10, (pp. 202-210). Carbondale,

IL: Southern Illinois University Press.

Dewey, J. (1923). The schools as a means of developing a social

consciousness and social ideals in children. Journal of Social Forces, 1,

513-517. Retrieved from https://www.jstor.org/stable/3005121

Dewey, J. (1923a). Outlawry of war: What it is and is not. Chicago, IL:

American Committee for the Outlawry of War.

Dewey, J. (1939/1988)] [1988]. Foreword to Johnson's Mars in civilian disguise! In J. A. Boydston (Ed.), The later works of John Dewey, Vol.

14, (pp. 355-356). Carbondale, IL: Southern Illinois University Press.

Dewey, J. (1971 rev.) How we think. Chicago, IL: Henry Regnery Co.

Ekirch, A. A. (1956). The civilian and the military. New York, NY: Oxford University Press.

Ferrell, R. E. (1953). Peace in their time: The origins of the Kellogg-Briand Pact. New Haven, CT: Yale University Press.

Levinson, S. O. (1918, March 9). The legal status of war. New Republic, 16, 171-173.

Levinson, S. O. (1918a, December 9, 1918). [Salmon O. Levinson papers].

Joseph R. Regenstein Library, University of Chicago, Chicago IL.

Limberg, M. (2014). In relation to the Pact: Radical pacifists and the

Kellogg-Briand Pact, 1928-1929. Peace \& Change, 39(3), 395-420. doi:

10.1111/pech.12079

Martin, J. (2002). The education of John Dewey: A Biography. New York,

NY: Columbia University Press.

Patterson, D. S. (1976). Toward a warless world: The travail of the American peace movement, 1887-1914. Bloomington, IN: Indiana University Press.

Ratner, J. (Ed.). (1929). Characters and events. Vol. II. New York, NY: Henry Holt \& Co. 
222 Howlett \& Cohan-Dewey: His role in public scholarship

Stoner, J. E. (1943). S. O. Levinson and the Pact of Paris. Chicago, IL: University of Chicago Press.

Westbrook, R. (1991). John Dewey and American democracy. Ithaca, NY: Cornell University Press.

Young, N. (2013). Concepts of peace: From 1913 to the present. Ethics and

International Affairs, 27, 157-173. doi: 10.1017/S0892679413000063

Dr. Charles F. Howlett. Professor, education división. Molloy College (USA)

Audry Cohan. Professor, education division. Molloy College (USA)

Contact Address: Acohan@molloy.edu 


\section{HSE}

Historia Social y de la Educación

Social and Education History
Hipatia Press

www.hipatiapress.com

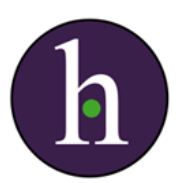

Instructions for authors, subscriptions and further details:

$\underline{\text { http://hse.hipatiapress.com }}$

1939: L'escola renovadora de Catalunya se'n va a l'exili

Salomó Marquès ${ }^{1}$

1) Universitat de Girona (Spain)

Date of publication: October $23^{\text {rd }}, 2016$

Edition period: October 2016 - February 2016

To cite this article: Marquès, S. (2016). 1939: L'escola renovadora de Catalunya se'n va a l'exili. Social and Education History 5(3), 223-245. doi:10.17583/hse.2016.2180

To link this article:http://dx.doi.org/10.17583/hse.2016.2180

\section{PLEASE SCROLL DOWN FOR ARTICLE}

The terms and conditions of use are related to the Open Journal System and to Creative Commons Attribution License (CC-BY). 


\section{9: L'Escola Renovadora de Catalunya se'n va a l'Exili}

Salomó Marquès

Universitat de Girona (Spain)

\section{Resum}

Durant la República, a Catalunya i arreu de l'Estat s'aconseguiren uns alts nivells d'instrucció i educació. Per bastir una societat més democràtica, justa i solidària les autoritats republicanes invertiren molt en educació. Es tractava d'ajudar els petits ciutadans a pensar críticament $\mathrm{i}$ viure els valors republicans. La cientificitat $\mathrm{i}$ l'ensenyament experimental eren la base de l'escola republicana. El salt qualitatiu que es va produir de l'escola de la monarquia a la de la República va ser notable.

Amb la victòria de les armes dels rebels a la República i la instauració de la dictadura franquista es va produir un trencament radical amb aquest model escolar. Molts dels mestres capdavanters se n'anaren a l'exili, sobretot a Mèxic i a altres països americans. Allà pogueren continuar ensenyant i practicant les metodologies renovadores que havien implantat a Catalunya. L'exili americà de tants bons mestres va comportar un enriquiment escolar als països d'acollida, mentre que l'escola sota la dictadura franquista reculava a models autoritaris i tradicionals.

Paraules clau: Escola republicana, exili del magisteri, renovació pedagógica, escoles en camps de concentració. 


\section{9: La Escuela Renovadora de Cataluña se va al Exilio}

Salomó Marquès

Universitat de Girona (Spain)

\section{Resumen}

Durante la República se consiguieron unos elevados niveles de enseñanza y educación en Cataluña y, en general, en España. Para construir una sociedad más democrática, justa y solidaria las autoridades republicanas invirtieron mucho en educación. Se trataba de ayudar a los pequeños ciudadanos a pensar de manera crítica y avivir los valores republicanos. Las bases de la escuela republicana eran la cientificidad y la enseñanza experimental. El salto cualitativo que se produjo de la escuela de la monarquía a la escuela republicana fue notable.

Con la victoria militar de los rebeldes a la República y la instauración de la dictadura franquista se produjo una radical separación de modelos escolares. Muchos de los maestros líderes de la renovación pedagógica marcharon al exilio, especialmente a México y otros países americanos. Allí continuaron enseñando y practicando las metodologías renovadoras que habían practicado en Cataluña. El exilio americano de tantos maestros excelentes condujo a una notable mejora escolar en los países de acogida, al tiempo que la escuela bajo la dictadura franquista retrocedía a modelos autoritarios y tradicionales.

Palabras clave: Escuela republicana, exilio del magisterio, renovación pedagógica, escuelas en campos de concentración. 


\section{9: The Reformist School of Catalonia goes to Exile}

Salomó Marquès

Universitat de Girona (Spain)

\section{Abstract}

During the Second Republic in Catalonia and throughout Spain, levels of education were high. With the aim of building a more democratic, fair and supportive society, Republican authorities invested in education. Their efforts focused on helping citizens think critically and live with Republican values. Science-based and experienced learning were also fundamental in Republican schools. The qualitative leap from schools in the Monarchy to schools in the Republic was significant.

The victory of fascism and the establishment of the Franco dictatorship, brought a radical break with the educational model developed. Many pioneering teachers were exiled to Mexico or other American countries. Where, they could continue to teach using the reform pedagogies they had previously developed in Catalonia. The American exile of so many teachers enriched schools in the receiving countries, while schools in the Franco dictatorship fell back into more authoritarian and traditional models.

Key words: Republican schools, teacher exile, educational reform, schools in concentration camps. 


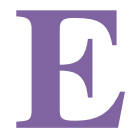

ncara se senten veus de líders polítics que, quan se'ls pregunta sobre la qualitat democràtica del país, fent referència als tics i actituds franquistes que es veuen pel territori i als mitjans de comunicació (només cal veure segons quines tertúlies), manifesten que ells no miren enrere. Un error enorme! perquè per mirar endavant, per avançar, és necessari saber d'on venim. I si mirem enrere ens adonarem que encara no hem fet cau i net d'una duríssima Guerra Civil amb vencedors i vençuts.

La mirada retrospectiva ens ha permès de conèixer la peripècia humana $i$ professional d'un col·lectiu que sempre ha tingut un paper important en la formació dels ciutadans del país, em refereixo als mestres. El text que segueix ofereix una panoràmica general del que va passar amb aquest col-lectiu que treballava a Catalunya durant la República; es presenta la peripècia d'aquells mestres, homes i dones, que van marxar a l'exili per no ser atrapats pels rebels, que van imposar una llarga i dura dictadura. Les dades que dono són el resultat de més de vint anys d'investigació, sobretot oral, per recollir el testimoni directe dels protagonistes (homes i dones). Als arxius oficials no hi havia dades dels mestres exiliats. Per a les autoritats franquistes, aquests homes $\mathrm{i}$ dones que havien treballat amb entusiasme $\mathrm{i}$ dedicació format les joves generacions no existien.

El fet que Catalunya sigui un territori de frontera va afavorir que el mes de gener i els primers dies del febrer del 1939 milers de republicans passessin la ratlla i entressin a França. Entre ells, molts mestres. En altres territoris de 1'Estat els mestres (i els republicans en general) no tingueren tanta sort i van ser assassinats directament per falangistes o condemnats per tribunals en judicis sumaríssims.

\section{L'Ensenyament a Catalunya durant la República}

\section{La República en Pau}

Per poder valorar plenament el que va representar l'exili del magisteri de Catalunya cal conèixer la política educativa de la República; la -que el govern central i la Generalitat van dur a terme durant el temps de pau (abril 
de 1931 - juliol de 1936) i els de revolució i guerra (juliol de 1936 - febrer de 1939). En destaco el fets més rellevants.

Durant els primers anys republicans, l'anomenat bienni progressista, el govern de l'Estat i el català coincideixen en una política de millorar l'educació. Invertir en educació era un dels camins indispensable, segons les autoritats polítiques de Madrid i Barcelona, per fer un país més just, més solidari, més europeu. Es tractava d'ajudar els infants a passar de súbdits a ciutadans.

Per primera vegada a la història d'Espanya, el ministre d'Instrucció Pública va ser un mestre, Marcel-lí Domingo. Mestre i polític nascut a Tarragona, va ser fundador del Partit Radical Socialista. No havien passat encara dues setmanes de la proclamació de la República que el govern provisional promulgava el Decret sobre la Llengua (29 d'abril de 1931), que afavoria l'entrada del català a l'escola, després d'anys de repressió de la Dictadura de Primo de Rivera; també promulgava el decret sobre coeducació. Domingo va instituir les "Misiones Pedagógicas" per fer arribar la cultura a les poblacions més abandonades del país.

A més, s'aprovarà el pla de construccions escolars per eliminar l'enorme dèficit existent; faltaven més de 27.000 escoles arreu de l'Estat! El govern establirà un pla de creació de 7.000 escoles el primer any i 5.000 els anys següents, fins a eixugar el dèficit. Per altra part, s'intensificaran les ajudes als mestres perquè surtin a l'estranger per conèixer experiències escolars en altres països d'Europa: com ara França, Suïssa, Bèlgica, etc.

I, el que és més important, el 1931 es promulgarà un nou pla de formació de mestres, el Pla Professional, que millorava qualitativament el pla existent de l'any 1914. El nou pla tenia una durada de quatre anys: els tres primers de formació teòrica; i el quart, de pràctiques pagades. Els continguts teòrics els configuraven tres grans blocs. Per una part, les matèries que ajudaven a la formació personal dels futurs mestres (filosofia, pedagogia, psicologia, història...); en un segon grup hi havia les matèries del programa escolar, les assignatures de matemàtiques, llenguatge, geografia, ciències naturals, etc.; el tercer bloc era el de la didàctica d'aquestes matèries. No n'hi havia prou de saber bé els continguts que caldrà ensenyar a l' escola, sinó que el que interessava era saber ensenyar bé aquestes matèries, i fer-ho desvetllant l'interès dels infants. Una vegada aprovats aquests tres cursos, els futurs mestres passaven un any de pràctiques en una escola per aprendre 


\section{Marquès - 1939: L'escola renovadora de Catalunya}

directament la feina al costat d'un mestre expert. Si aprovaven en acabar aquests quatre cursos, ja tenien feina assegurada.

El Pla Professional mostrava clarament la voluntat política d'invertir en educació per millorar la societat; es tractava de preparar els futurs mestres perquè fossin motor de canvi.

Les paraules del director general de Primer Ensenyament mostren la voluntat política de les autoritats republicanes: "Borrar la desigualdad más irritante, más injusta y más perturbadora que existe; la desigualdad ante la cultura. Necesitamos una escuela primaria para todos, enseñanza secundaria y universitaria no para los ricos sino para los capaces, sean ricos o pobres. Una cultura postescolar para los que no reciben la enseñanza superior y una preparación suficiente para todos los empleos de la agricultura, la industria y el comercio".

Per la seva part, el govern català recuperarà les Escoles d'Estiu, un espai excepcional de formació permanent del magisteri, que ja s'organitzaven durant la Mancomunitat i que Primo de Rivera va prohibir. En aquestes trobades voluntàries, celebrades a Barcelona, a més dels diversos cursos que s'oferien per a l'actualització del mestres, es van debatre els grans temes relacionats amb l'escola. El 1932 es tractà el tema "Problemes que planteja l'ensenyament del llenguatge a Catalunya», a partir del decret sobre la llengua promulgat pel govern l'abril del 1931; l'estiu següent, «L'educació moral i cívica a les escoles de la república», i el 1934, «L'escola unificada: mitjans per establir-la». Es tractaven els temes de fons del nou model escolar republicà.

Els participants a les escoles d'estiu treballaven les metodologies relacionades amb el moviment de l'Escola Nova que es practicaven en països europeus i, també, a Catalunya: Montessori, Decroly, Freinet, etc., en alguns casos, amb la participació directa dels mateixos autors. A més, al llarg de l'any es feien "Conferències pedagògiques" en capitals de comarca organitzades per les associacions de mestres, i cursos de reciclatge de català amb el mestratge de Pompeu Fabra, a les Escoles Normals per als futurs mestres, i cursos per als mestres en exercici. Les aportacions de material pedagògic i de llibres escolars i de lectura per part de diferents editorials de Barcelona contribuïren a crear el clima que feia realitat el lema «Els escolars, els primers ciutadans de Catalunya» i que permetien parlar de la «República dels mestres». 
A més, la Generalitat va crear a Barcelona una nova Escola Normal de Mestres amb el concepte d'assaig pedagògic per respondre «a les modalitats típiques de la seva fesomia catalana» ${ }^{1}$. Això li permeté de formar un claustre de professorat excel·lent amb un funcionament més àgil i democràtic que el de les Escoles Normals estatals. Era una Normal que volia respondre «a les modalitats típiques de la seva fesomia catalana».

Aquest programa polític es va portar a terme durant els anys republicans de pau i va estar condicionat pels canvis polítics. No hi hagué el mateix interès per part de les autoritats polítiques durant el bienni progressista, quan governaven forces renovadores, que durant el bienni negre (novembre de 1933 - febrer de 1936) quan el poder va estar en mans dels sectors conservadors.

Juntament a la política escolar i educativa de les autoritats governamentals, hem de destacar que força escoles públiques i algunes de privades van implantar metodologies $i$ pràctiques renovadores. Les associacions de mestres i força mestres s'esforçaren per garantir una bona escola per formar els futurs ciutadans. A partir d'ensenyaments centrats en l'observació i l'experimentació, de desvetllar l'interès dels nois i noies, de trencar les parets de l'escola i fer activitats a l'aire lliure, de fomentar intercanvis escolars, de publicar revistes, etc., un ampli sector del magisteri va treballar amb entusiasme i convenciment per bastir una nova societat. Al costat de la voluntat política dels governants espanyols i catalans, hi havia la pràctica pedagògica de molts mestres arreu del país. Serà durant aquests anys republicans que l'ensenyament aconseguirà els nivells més elevats de qualitat pedagògica, gràcies a la dedicació dels mestres i a la política de les autoritats de Catalunya i de l'Estat (González-Agàpito, Marquès, Mayordomo i Sureda, 2002).

\section{Temps de Revolució i Guerra. El CENU}

El clima sociopolític canviarà radicalment el juliol del 1936 amb l'aixecament contra la República. A Catalunya els rebels van ser derrotats i s'inicià una etapa de revolució i de guerra arreu de l'Estat. A finals del mateix mes de juliol es crearà a Barcelona el Consell de l'Escola Nova Unificada (CENU) perquè «la voluntat revolucionària del poble ha suprimit l'escola de tendència confessional. És l'hora d'una nova escola, inspirada en 


\section{Marquès - 1939: L'escola renovadora de Catalunya}

els principis racionalistes del treball i de la fraternitat humana. Cal estructurar aquesta escola nova unificada, que no solament substitueixi el règim escolar que acaba d'enderrocar el poble, sinó que creï una vida escolar inspirada en el sentiment universal de solidaritat i d'acord amb totes les inquietuds de la societat humana i a base de la supressió de tota mena de privilegis» ${ }^{2}$.

La realitat escolar canvià de manera radical. Les escoles de les congregacions religioses, masculines i femenines, van tancar; molts frares i monges hagueren d'abandonar els col-legis per salvar la vida; d'altres van ser assassinats. El govern català confiscà els seus edificis, que majoritàriament continuaren dedicats a l'ensenyament; això sí, amb un nou professorat i amb noms canviats, relacionats amb la nova situació revolucionària. Per exemple, a Girona l'escola dels germans de La Salle s'anomenarà Carles Marx, la dels germans Maristes serà la Durruti, etc.

Els nens i nenes aniran plegats a la mateixa escola; per fi es farà realitat arreu del territori l'escola mixta, coeducativa, que tants recels havia despertat en sectors conservadors. Hi haurà mestres que deixaran l'escola per anar al front a defensar la República; d'altres seran cridats a files durant aquest període de guerra; començaran a arribar infants d'altres indrets de l'Estat fugint de la situació de guerra; ben aviat hi haurà bombardejos -i aniran en augment-, etc. Tot i que es viurà una situació anòmala, això no privarà que les autoritats continuïn creient en la importància de l'educació. Serveixi d'exemple el cas de Girona, on el mes de juliol del 1938, quan la situació bèl-lica ja estava decantada a favor dels rebels, les autoritats municipals, amb la presència del president Companys, encara van posar la primera pedra d'una nova escola concebuda com un palau de cultura! (Fontquerni i Ribalta, 1982).

El 10 de febrer del 1939 les tropes rebels arribaran a la frontera amb França. S'ha acabat la República a Catalunya! Començarà una llarga i dura dictadura en un país amb vencedors i vençuts. Les intencions de les noves autoritats eren molt clares: «(...) A los otros, a los del "hecho diferencial", nuestra notificación de que han sido vencidos por la fuerza de las armas, y que si no quieren ser hermanos de los otros españoles les impondremos la ley del vencedor, porque nosotros, los combatientes, al terminar la guerra en Cataluña damos también por terminados y para siempre los hechos 
diferenciales.» Aquestes van ser les paraules del comandament militar, el tinent coronel de la legió Alonso Vega, a la seva entrada a Olot.

\section{Cap a l'Exili}

El fet que Catalunya sigui un país de frontera va afavorir que molts republicans marxessin cap a França el gener i primers dies de febrer del 1939. Es calcula que gairebé mig milió de republicans entraren al país de la «Liberté, egalité, fraternité». Ho feren majoritàriament per la Jonquera i Portbou; de totes maneres, qualsevol camí va ser bo per sortir del país i no ser atrapat per l'exèrcit rebel (coll d'Ares, coll de Banyuls, coll de Lli, coll de la Manrella, portella de Mantet, Tapis, Puigcerdà, etc.).

L'entrada a França no va ser fàcil. El govern francès no va acollir els fugitius amb el braços oberts. La frontera francesa estava tancada. Després d'unes negociacions s'obrí per deixar passar les dones i els infants, després els malalts i, finalment, la resta (civils i soldats). No fa pas gaire que el primer ministre francès, Manuel Valls, així ho va reconèixer ${ }^{3}$. Marxaren derrotats i barallats; la gran majoria van ser conduïts als camps de concentració de les platges d'Argelers, Sant Cebrià, el Barcarès; més tard, cap a l'interior: Ribesaltes, Agde, Bram, etc. ${ }^{4}$ Les autoritats polítiques republicanes, militars $\mathrm{i}$ una minoria es van estalviar aquest calvari vergonyós.

Entre aquests milers de refugiats hi havia una bona colla de mestres. Més de 560 mestres que treballaven a Catalunya durant els anys de la República (en pau i en guerra) sortiren del país (Marquès, 1995). Una xifra que representa aproximadament l' $11 \%$ del col·lectiu del magisteri que hi havia a Catalunya. Per sexes, hi havia aproximadament un $26 \%$ de dones i un $74 \%$ d'homes.

Aquest col-lectiu, políticament i pedagògicament, era plural. N'hi havia que militaven en el POUM, el BOC, AC, el PSUC, ERC, la USC, en l'anarquisme, etc.; d'altres «només» eren republicans i/o nacionalistes. Alguns tenien càrrecs municipals (alcaldes o regidors), també n'hi havia que eren diputats al Parlament de Catalunya o tenien càrrecs en el govern espanyol. Alguns van ser milicians de cultura al front de guerra. N'hi havia que practicaven les tècniques Freinet, d'altres promovien escoles racionalistes; alguns eren entusiastes de les propostes decrolynianes; molts 


\section{Marquès - 1939: L'escola renovadora de Catalunya}

practicaven tot tipus de propostes del moviment de l'Escola Nova, etc. La gran majoria eren mestres en escoles públiques; alguns treballaven en acadèmies privades. També hi havia professors i directors d'Escoles Normals, membres del Consell de l'Escola Nova Unificada (CENU), inspectors de primer ensenyament. El col-lectiu de mestres exiliats el formaven majoritàriament líders de la renovació pedagògica.

\section{A França}

Entrats a França, n'hi haurà que tornaran aviat cap a casa, on les autoritats franquistes els depuraran i castigaran (González-Agàpito i Marquès, 1996) La gran majoria dels que es queden a França seran internats en camps, però tindran sempre l'objectiu de sortir al més aviat possible, amb ajuda o escapant-se. Des del punt de vista cultural s'organitzaran els «barracons de cultura», amb participació directa dels mestres. Es tractava d'oferir activitats culturals de tot tipus: conferències, classes d'alfabetització, classes de francès, música, etc., per evitar l'ensorrament moral, el desànim, la depressió. Aquest tipus d'activitats s'estengueren a la majoria de camps: Argelers, Barcarès, Gurs, Agde, etc.

En algun també hi haurà escoles per als nens i nenes. Al camp de Sant Cebrià hi va haver una escola mixta, l'escola Canigó, amb sis graus i un preescolar. Ramon Moreno, un dels mestres gironins que hi va treballar, recordava que estava instal-lada en barracons de fusta, amb un espai dedicat a aules $\mathrm{i}$ un altre que servia de dormitori per als mestres (una situació privilegiada de la qual no gaudien la majoria dels refugiats). Amb permís de les autoritats militars, algunes vegades van sortir fora dels límits del camp per recollir material per a les classes de botànica (!), tal com acostumaven a fer a les escoles dels seus pobles. Aquesta experiència va durar vuit mesos (Marquès, 2002).

El mestre lleidatà Josep Vilalta va treballar a l'escola del camp de Bram, a la regió de l'Aude. Ho recorda a les seves memòries: «En el camp de concentració no només hi havia homes sinó també dones i molts nens. Per a aquests s'havia creat una espècie d'escola que funcionava al final del camp. Jo, com a mestre, vaig ser cridat a fer-me càrrec d'un grup de nens. El director era un refugiat madrileny d'uns seixanta anys. Em vaig presentar $\mathrm{i}$ se'm va destinar a un barracó on hi havia un grup de nens entre 12 i 15 anys. 
El material de classe de què disposaven era una llibreta i un llapis.» En alguns camps editaren la revista Profesionales de la Enseñanza. Els sindicats francesos els ajudaven amb llibres, paper, màquines d'escriure, pilotes, etc. (Cruz, 1994a).

Al camp d'Agde es feien classes de francès, anglès i català. També s'hi ensenyava història i geografia de Catalunya. De fet, aquest camp es va anomenar «el camp dels catalans» pel fet que la majoria dels internats eren de Catalunya i els rètols del camp i les ordres per l'altaveu es feien en català; també hi havia una coral i una companyia de teatre catalanes.

De totes maneres, ben aviat la vida als camps es veurà condicionada per l'ocupació nazi i la situació de guerra que viurà França. Alguns dels mestres que aconseguiren sortir dels camps s'incorporaran a la Resistència lluitant contra els ocupants; d'altres seran obligats a incorporar-se als batallons de treballs forçats, que sovint van ser enviats al nord, a construir les defenses militars. Una bona colla procuraran marxar del país cap a Amèrica amb l'ajuda d'organismes internacionals i d'institucions republicanes espanyoles com el SERE (Servicio de Evacuación de los Republicanos Españoles) i la JARE (Junta de Auxilio a los Refugiados Españoles), que estaven enfrontades a causa de divisions polítiques.

\section{Cap a Amèrica}

A tots els països d'Amèrica hi haurà presència de mestres republicans exiliats. El fet que la llengua de relació fos l'espanyola va afavorir la tria de país. Ho veurem més endavant. Hi ha tres països que mereixen una atenció especial. Per una part, Mèxic i Veneçuela, on la presència de mestres va ser més nombrosa. En el cas de Mèxic, per raons òbvies, ja que va ser l'únic país que va obrir les portes de bat a bat als republicans. Allà s'hi establí el govern de la República a l'exili.

L’altre país que mereix una atenció especial és la República Dominicana, governada pel dictador Trujillo. Els republicans marxen del seu país per no caure en mans de Franco i són rebuts a Santo Domingo per un altre dictador. Possiblement Trujillo els acull per millorar la seva imatge internacional i per altres raons ${ }^{5}$. De totes maneres, el tracte que van rebre a l'illa no té res a veure amb el que els van dispensar altres països americans. El mestre lleidatà Antoni Mónico va treballar de pagès, a la República Dominicana, 


\section{Marquès - 1939: L'escola renovadora de Catalunya}

«perquè era de les poques possibilitats que teníem de viure; estava en una colònia de treball i quasi no teníem res per menjar. Hi havia la possibilitat de posar-te per lliure, però era molt difícil» (Marquès, 2003, p. 125). Aquest mestre marxarà el 1944 a Veneçuela. D'altres col·legues marxaran per anar a Cuba, Mèxic... De fet, quan va acabar la Segona Guerra Mundial la majoria de mestres (i de republicans) ja havien abandonat la República Dominicana.

\section{Mèxic}

El país que va acollir més mestres (i republicans) va ser Mèxic. Ja el 1937, en plena guerra, havia arribat una primera expedició de nens i nenes, la dels «Nens de Morelia» (Pla, 1985). De totes maneres, serà a partir del 1939 que des de França es promouran expedicions de republicans, sovint amb l'ajuda d'organismes internacionals com ara els quàquers. El Sinaia, amb 1.500 refugiats, va ser el primer vaixell a arribar a Veracruz. El diari ciclostilat que es publicava durant la travessa permet de veure com preparaven els refugiats explicant-los com era el país que els acollia. D'altres vaixells amb refugiats cap a Mèxic van ser Mexique, Ipanema, Cuba... També en sortiran cap a d'altres països. Winnipeg, Flandre, Orinoco, De Grasse, Champlain, són alguns dels noms més emblemàtics dels vaixells que van portar exiliats cap a Amèrica (Malgat, 2013, p. 93).

Des del punt de vista educatiu i escolar, podem distingir una triple presència de mestres exiliats a Mèxic (Cruz Orozco, 2004). Per una part, els que es quedaren a la capital federal. Aquests treballaran en els centres escolars creats pel govern republicà a l'exili: l'Instituto Luis Vives, el Colegio Madrid, l'Academia Hispano-Mexicana, el Colegio Ruiz de Alarcón. De fet, l'ensenyament en aquests col-legis era una repetició del que feien a Catalunya adaptat a la legislació mexicana. El gironí Marcel Santaló, parlant de les metodologies renovadores que aplicaven en aquests centres, manifestava:

"En el Vives, todos los maestros habíamos pertenecido a la Institución Libre de Enseñanza. Sus ideales consistían en ir formando un temperamento liberal. Formar hombres que pensaran que otro hombre puede tener razón en lo que piensa y en lo que dice, pues es la esencia de la democracia. Otro ideal sería el de buscar sentido a la vida. ¿Cuál 
es el objetivo de vivir? Si el objetivo de vivir es personal o bien fomentar la solidaridad u ocuparse de la comunidad toda. En la Institución se daba una mayor importancia a la formación espiritual". (Reyes, 1982, p. 191).

L'estil d'ensenyar dels mestres republicans era diferent del que empraven els mexicans a les escoles. La mestra berguedana Estrella Cortics, que va ensenyar durant la República a la ciutat de Barcelona, ho explicava d'aquesta manera: «En cuanto empecé a trabajar noté la primera diferencia entre las escuelas de España y las de aquí, y era que en México nos daban un programa que teníamos que cumplir, los maestros no teníamos independencia para hacer una cosa un poco más personal; además, como en los exámenes los alumnos tenían que contestar las preguntas del programa, pues teníamos que cumplirlo. Por otro lado, siempre me llamó la atención el que los maestros de la escuela no nos reuníamos para conversar sobre los problemas de la escuela o de los alumnos, y si nos reuníamos, era para hablar del sueldo, para hablar de los dineros.» (Marquès, 2001, p. 31).

Fora de la capital federal hi havia la xarxa de col-legis Cervantes. El Patronato Cervantes, presidit per Roura Parella, gràcies a les aportacions econòmiques del Comitè Tècnic del SERE, va afavorir la creació de col-legis a diferents ciutats: Córdoba, Jalapa i Veracruz (Veracruz), Torreón (Cuahuila), Tampico (Tamaulipas), Tapachula (Chiapas), Mérida (Yucatán), Cuernavaca (Morelos), etc. Eren centres que funcionaven autònomament; havien rebut diners per crear el col-legi per part del govern republicà, per iniciar les seves activitats, i a partir d'aquesta ajuda inicial havien d'espavilar-se.

A la premsa mexicana dels anys 1939 i 1940 s'hi troben informacions com aquesta: «Se abre concurso entre los profesionales de la enseñanza españoles exiliados en México para la provisión de plazas de directores de las escuelas primarias que organiza en México, D.F. y en algunas capitales de estados de la república este Patronato. Los aspirantes deberán presentar sus instancias, a las que acompañarán relación de méritos, en la Oficina de Trabajo del Comité Técnico de Ayuda a los españoles en México, Sinaloa, 56. El plazo de admisión de instancias terminará el día 27 de los corrientes y el 30 para los residentes fuera del D.F. Será condición preferente la de haber ejercido, en España, los cargos de director de escuela graduada de más de 


\section{Marquès - 1939: L'escola renovadora de Catalunya}

seis grados, inspector de Primera Enseñanza y profesor numerario de Escuela Normal.» En l'actualitat encara funciona el Colegio Cervantes de Torreón, dirigit per Antonio Méndez Vigatà, nét del fundador, el mestre lleidatà Antoni Vigatà Simó, membre d'una nissaga de mestres (Marquès, 2011). Aquest, igual que altres col-legues, a més de treballar al col-legi escrivien a la premsa local sobre temes educatius. Per exemple, Vigatà va publicar «El Método Decroly» i «La imprenta en la escuela» a El Siglo de Torreón, el 2 d'abril i el 12 de maig de 1940, respectivament. En aquests col-legis Cervantes, a diferència dels de la capital federal, l'alumnat era majoritàriament mexicà.

Fa uns anys, el mestre Antoni Bargés, que treballava al Colegio Cervantes de Córdoba, a l'estat de Veracruz, m'explicava com feia les classes i de quina manera anaven incorporant les metodologies renovadores a l'escola. Em deia, ja jubilat: «Avui dia encara trobem força antics alumnes pel carrer que ens recorden, i és que hem estat mestres diferents dels d'aquí. Jo els deia: "Us heu d'interessar per la premsa de Mèxic, saber el que passa a Mèxic, quines relacions té Mèxic amb la resta de països." I els altres mestres arribaven: "Doncs bé, anem a dictar la lliçó, tal, tal, tal." Jo sempre, entorn de la lliçó que explicava, sempre tenia alguna novetat per explicar. De manera que nosaltres érem diferents dels altres mestres en aquest sentit.» Els seus antics alumnes em recordaven que la tarda dels divendres solia projectar diapositives a l'aula, i com al pati acostumava a fer amb la sorra mapes en relleu per destacar els rius, volcans i muntanyes de la zona... Una pràctica, aquesta d'ensenyar la geografia en relleu, que ja havien practicat força mestres a Catalunya els anys de la República (Marquès, 2004).

Un tercer grup de mestres el formaren els mestres exiliats que van treballar fora dels col-legis dirigits $\mathrm{i}$ pagats pel govern republicà. Eren mestres amb experiències relacionades amb el camp de l'educació popular. Els noms de Patricio Redondo, José de Tapia i Ramon Costa Jou (Lozano, 1999, p. 109), mestres freinetistes que havien treballat amb entusiasme i dedicació a Catalunya i que ara ho continuaran fent a Mèxic, en són l'exponent més clar. L'ensenyament d'aquests tres mestres estava orientat directament als nois i noies mexicans i no pas als fills dels exiliats republicans. L'estil de treball l'explica Tapia amb aquestes paraules: 
"Nos basábamos en la educación por el trabajo espontáneo, en la pedagogía del tanteo, del vas bien y si no rectifica, en la formación de niños libres y responsables, de criaturas impregnadas de amor por la vida. Procurábamos despertar en nuestros chicos el apetito por el conocimiento de aspectos explicativos de la realidad que, a la vez, les permita actuar sobre ella. En cuanto les da sed, no hay nada ni nadie con capacidad para detenerlos". (Marquès, 2001, p. 34).

Les paraules de la mestra Estrella Cortics resumeixen molt bé el que va ser, en general, la feina professional dels mestres republicans:

"Para mí, la aportación fundamental de los españoles en México fue el ansia de trabajo serio, de honradez, de formalidad. Creo que fue un ejemplo y gracias a esto, los españoles nos pudimos defender aquí. En la esfera intelectual vinieron muchos de una gran talla que, lógicamente, se tenían que notar aquí, donde hacían falta en muchas ramas, además estos formaron a otros en sus teorías, en su manera de trabajar y no es fácil que esto se pierda”. (Lozano, 1999, p. 120).

\section{Veneçuela}

Veneçuela va ser l'altre país americà on van anar a raure més mestres catalans exiliats. Sobretot a la capitat, Caracas, i a Maracaibo. En aquest país també hi havia col-legis fundats per exiliats bascos que compartien amb els exiliats catalans el republicanisme, el nacionalisme (català o basc), les metodologies renovadores, etc. La diferència més notable entre els mestres catalans $\mathrm{i}$ els bascos era el fet que a les escoles amb mestres catalans i espanyols l'ensenyament era laic, com ho era l'escola al seu país durant la República, mentre que a les basques hi havia força pràctiques religioses (misses, primeres comunions, etc.). Els col-legis bascos tenien un toc d'educació religiosa molt notable; de totes maneres aquesta orientació religiosa no els privava de ser declaradament antivaticans, ja que el Vaticà havia donat suport a Franco (Marquès i Martín Frechilla, 2002, p. 76).

A Caracas, el 1940, Bartomeu Oliver, juntament amb la seva esposa, Dolors Jordana, va crear l'Institut-Escola La Florida, seguint l'estil pedagògic renovador que tenien els instituts-escola de Madrid i de Barcelona, concebuts com a centres pilot i experimentals. A la mateixa 


\section{Marquès - 1939: L'escola renovadora de Catalunya}

capital, el matrimoni format per Joan Gols i Mercè Cavagliani, també el 1940, creà 1'Institut Montessori-San Jorge. El nom ja deixa clars els dos eixos del centre: renovació pedagògica a partir de l'aplicació de la pedagogia de la doctora italiana i la procedència catalana dels seus fundadors. En aquest centre hi treballaren mestres catalans $i$ alguns procedents d'altres indrets de l'Estat. Igual que a Mèxic, el claustre d'aquests col·legis els primers temps el conformaven mestres exiliats. Amb el pas del temps s'hi anà incorporat professorat autòcton. En ser escoles privades, era la direcció del centre que escollia el professorat; en aquest aspecte, les relacions i els coneixements fets a Catalunya van ser un bon camí per trobar feina i ajudarse mútuament.

Hi ha algun cas de mestres no catalans que treballaren a Catalunya durant la República. És el cas del Colegio Leal. El seu director, Luis Leal, professor de 1'Escola Normal d'Oviedo durant els primers anys republicans, va ser director de la Normal de Girona durant els anys de guerra. I la seva esposa, Pilar Munarriz, també professora al mateix col-legi, va ser inspectora a Girona durant la República. Des de Girona, el matrimoni Leal-Munarriz marxarà a França i posteriorment arribarà a Veneçuela, on continuarà dedicant-se a l'ensenyament fins a la seva jubilació ${ }^{\text {. }}$.

A Maracaibo, capital de l'estat de Zuila, el matrimoni Barrull-Zavala, ell català i ella basca, que havien treballat en escoles rurals de Catalunya durant la República, treballaran en diferents àmbits escolars a 1'exili. Primer en el de la formació del magisteri, després en el dels infants abandonats, i finalment crearan el seu propi centre, el Colegio Cervantes, que ensenyava des del jardí d'infants fins a l'entrada a la universitat. Josep Barrull, militant del PSUC i membre de la maçoneria, fill de la Granadella, va entrar amb l'exèrcit republicà a França. La basca Carolina Zavala, que exercia de mestra a la Granadella, on va conèixer el seu futur marit, marxarà a l'exili francès $i$ allà es retrobarà amb Barrull. Amb l'ajuda del SERE marxaran des de Bordeus cap a Maracaibo.

La parella mantindrà al llarg dels anys una profunda convicció de la dimensió social de la feina docent. Tal com feia a Catalunya, Barrull no deixarà d'escriure a la premsa local sobre temes educatius. L'any 1997, amb motiu de l'homenatge que li van fer els antics alumnes i del reconeixement públic per part de les autoritats, defensava el compromís social del mestre al diari Panorama de Maracaibo amb aquestes paraules: «Un maestro tiene que 
ser de vocación, no tiene que pensar en una profesión como medio de lucrarse, porque esta profesión no es para eso. Es para servir a los demás y es para engrandecer al país y tiene que entregarse así, como creo que en algunos momentos me he entregado. Quizás los jóvenes de hoy tienen otro criterio, pero creo que es difícil orientar la educación porque se tiene que basar en la completa libertad, y la educación privada está cada vez más floreciente, cada vez aumenta más y este tipo de educación no creo que sea la mejor. Se tendría que buscar una educación de estado, generalizada, con programas bien establecidos, con orientaciones bien definidas, con una política educativa determinada.» (Marquès i Martín Frechilla, 2002, p. 139). Aquest convenciment de la dimensió social del mestre que ja els esperonava a Catalunya ho continuarà fent a l'exili americà.

Un altre nom destacat de l'exili veneçolà és el del mestre Raimon Torroja. A Catalunya, durant els anys republicans, va ser el director de 1'escola annexa a la Normal de la Generalitat. Professional ben preparat, participà a les escoles d'estiu i en activitats de formació de professorat. Va publicar nombrosos llibres, tots de caire educatiu; un dels més importants va ser Cartilla de civisme i dret. Abans de la República va ensenyar a 1'escola d'Arenys de Munt. En un informe a l'Ajuntament en què demanava millores materials a l'escola (millorar la ventilació, pintar de colors adients les aules, etc.) feia també aquesta demanda a les autoritats municipals: «També cal treure la tarima del professor, per antiestètica i antipedagògica.» Una declaració d'intencions de quin era el paper del mestre: no pas la persona que parla des de dalt de la tarima, sinó aquell que és al costat dels infants acomboiant-los en el creixement personal.

Després de sortir de la presó Model, on havia estat tancat pels franquistes, no podrà resistir la pressió sociopolítica del nou règim, de la dictadura, i el 1948 marxarà a Veneçuela via Nova York, atenent la invitació d'altres companys mestres: H. Almendros des de Cuba, T. Bartrolí i J. Virgili des de Caracas. Una mica més tard hi arribarà la família: l'esposa, els tres fills i la tia, Coloma Torroja, que també era mestra. A la capital de Veneçuela ja l'esperaven altres companys exiliats i comença a treballar al Colegio América. En va ser professor i subdirector fins a la seva jubilació. A més, farà conferències de marcat caràcter educatiu a la Universidad del Aire de Radio Caracas. El seu cas és un exemple d'exili tardà, però no és l'únic. 


\section{Marquès - 1939: L'escola renovadora de Catalunya}

Poques setmanes abans de marxar a l'exili, escriurà una carta a una seva antiga alumna en la qual, entre altres coses, li manifesta: «Segueix per aquest camí i perfecciona la forma, és a dir, el lèxic, ortografia i sintaxi, amb preocupació constant, mai amb deixadesa, que sempre que surti una carta de les teves mans tingui el nivell de perfecció que la teva edat i coneixement puguin donar-li. (...) Pensar bé el que vas a escriure, comprovar si és correcte i delicat, si pot ofendre, si és discret; i expressar-ho immediatament amb el llenguatge més correcte i breu. (...) No et desanimis mai. Has d'arribar a ser una dona de carrera i apta per crear-te una posició independent. Els temps canvien i exigeixen això de les dones.» Paraules d'estímul a una noia que contrasten amb la situació humiliant que patien moltes dones sota la dictadura franquista, on imperava el model imposat per la Secció Femenina de dona obedient i submisa a l'home.

Joan Campà Claverol és un altre nom destacat entre els mestres exiliats. Va ser un dels pocs mestres exiliats d'ideologia $i$ activa militància anarquista. Educat a 1'Escola Natura de Barcelona, dirigida per Puig i Elias, durant la República treballarà a l'Escola Ferrer i Guàrdia, de Terrassa. Quan va esclatar la revolució el juliol del 1936, va formar part del Consell de 1'Escola Nova Unificada (CENU) i va ser secretari general del Sindicat de Professions Lliberals; va elaborar l'avantprojecte del decret sobre la llengua. Ocupada Catalunya, marxarà a l'exili, a París; el desembre del 1939 embarcarà a Bordeus cap a la República Dominicana. A causa de les tensions amb els comunistes espanyols exiliats, decideix marxar a Veneçuela.

Treballarà a diferents nivells educatius fins a la seva mort el 1991. Primer va ser professor d'agricultura en una escola rural; després, mestre de dibuix i treballs manuals a les escoles federals del Ministerio de Educación; professor a l'Instituto La Florida; després, al Colegio América. El 1956 fundarà l'Instituto Einstein.

Membre de l'Asociación Nacional de Institutos Educativos Privados (ANDIEP), en l'article sobre «Filosofía pedagógica de la asociación», escriu: «La filosofía educativa consistirá en tender a la formación del hombre; objetivo sólo realizable dentro de un ambiente humanístico. (...) La tendencia educativa de nuestra asociación será adaptar al hombre en su totalidad de espíritu y vida sin olvidar jamás la complejidad históricocultural de nuestra época y el medio nacional en que habrá de vivir. Nuestra 
educación habrá de convertirse en fuerza estimuladora para hallar la plenitud humana sin que se utilicen aislamientos ni mutilaciones. Rehuiremos siempre los dogmas y los sectarismos enervantes.»

En el camp polític, Campà va continuar actiu. Des de Veneçuela participarà en els congressos internacionals anarquistes celebrats a Amèrica $i$ a Europa. Continuarà actiu en la lluita antifranquista i, en alguns moments, participa en la vida política veneçolana. Va fundar el Centre Català a Caracas. Escriurà sobre educació al diari El Libertario de Caracas.

\section{Altres Països Sud-Americans}

Hem destacat les activitats dels mestres republicans exiliats a Mèxic i Veneçuela perquè són els dos països amb més presència de mestres en actiu (homes i dones) exiliats. De totes maneres, no són els únics països americans. De fet, des del Canadà fins a l'Argentina i Xile, passant pels països de l'Amèrica Central, és possible trobar mestres exiliats treballant en el camp de l'ensenyament. En alguns casos les seves aportacions van ser importants $\mathrm{i}$ feren contribucions positives en l'àmbit escolar i pedagògic del país d'acollida, fins i tot en alguns casos van tenir càrrecs de responsabilitat en el govern.

Recordem-ne alguns. Per exemple, Pau Vila Dinarès, que s'establirà a Colòmbia el 1939, fins al 1946, que marxarà a Veneçuela. Va ser professor a 1'Escola Normal de Bogotà, Colòmbia, combinant la seva tasca docent amb la científica. Una de les seves obres principals és Nueva geografía de Colombia. A Cuba hi haurà l'inspector Herminio Almendros, que a Catalunya va ser un dels impulsors de la pedagogia freinetiana; va publicar molts llibres escolars, i amb la victòria de la revolució castrista va col-laborar activament amb els nous governants; el 1959 va ser nomenat director general d'Educació Rural. També arribà a Cuba el 1943 Ramon Costa Jou, fugint de la República Dominicana; després passarà a Mèxic, i el 1960 tornarà a Cuba, convidat per les autoritats del país; del 1961 al 1967 va ser cap del Departament de Pedagogia de la Universitat de l'Havana.

A El Salvador el 1940 hi arribà la barcelonina Maria Solà i Ferrer per dirigir l'Escola Normal de Noies. El 1945 marxà a Guatemala a dirigir 1'Escola Normal de Noies, tot compartint docència a la Universidad de San Carlos. El 1954 marxarà, a causa del cop militar, s'establirà a Mèxic i 


\section{Marquès - 1939: L'escola renovadora de Catalunya}

ensenyarà a la Universidad Autónoma de México D. F. Serà una de les difusores de la pedagogia Waldorf a la Universidad de las Américas, a Puebla. A Xile, el 1940, hi arribà Dolors Piera, mestra lleidatana que juntament amb el seu pare formà part del grup Batec, que practicava $\mathrm{i}$ difonia la metodologia freinetiana. Arribà a Xile procedent de la República Dominicana, va treballar en una escola pilot privada, Bialik, de la comunitat jueva. Del 1949 al 1953 va ser assessora del Ministeri d'Educació. Fora del camp escolar, però en el marc educatiu, va treballar a favor de les dones $\mathrm{i}$ organitzà el I Congreso de Mujeres de Santiago. La lluita antifranquista va ser una altra de les seves constants (Soler, 2015, p. 187).

Seguint les referències d'aportacions de dones mestres a l'exili, hem d'esmentar la barcelonina Dolors Canals i Farriols, exiliada primer a Santo Domingo, posteriorment a l'Havana i, finalment, als Estats Units. Va treballar activament en el camp de l'educació maternal, al departament de Salut i Educació de Nova York (Soler, 2015, p. 221).

\section{I mentrestant, a Catalunya i Espanya...}

Força dels mestres exiliats acollits als països americans continuaren dedicats a l'ensenyament. I ho feren aplicant aquelles pedagogies renovadores que ja havien practicat a les escoles de Catalunya els anys republicans, en pau i en guerra. A les escoles americanes on treballaren tingueren fama ben guanyada per la qualitat de la docència i per l'estil de l'ensenyament. Hem de parlar d'una aportació positiva a l'educació i a les escoles als països d'acollida.

Mentrestant, a Catalunya (i a l'Estat Espanyol), el país que havien hagut de deixar per no caure en mans dels rebels a la República, s'imposava una dictadura que consolidava un país amb vencedors i vençuts. En el camp educatiu es recuperà el model d'escola tradicional i catòlica. Només cal recordar la resposta de l'inspector en cap de Girona a un mestre quan, el 1939, li demanava ajuda material per a la seva escola, en la qual faltava de tot. L'inspector li va respondre que en aquells moments el que calia era que a l'escola hi hagués mestres que fossin «más santos que sabios».

Les paraules del mestre de Salt, el setembre del 1939, quan deixà 1'escola, són significatives del nou clima escolar de la dictadura: «Año de la Victoria. Unas palabras: Después de tres años justos de dominio rojo, la 
escuela puede empezar el curso al amparo del Generalísimo Franco, Caudillo de España, que con sus ejércitos gloriosos ha redimido palmo a palmo el terreno de nuestra patria. Salt, nombre de fuerte prestigio por los innumerables asesinatos cometidos por sus hombres, experimentará, gracias al esfuerzo de los hombres de la nueva España, un cambio radical de vida. El nombre de Dios presidirá ya para siempre estas escuelas. Y tú, maestro que me sucedes, recuerda que España espera de tu abnegado sacerdocio la regeneración de estos niños, su educación esmerada y su acercamiento hacia el Todopoderoso, con su corazón rebosante de amor hacia Él y al prójimo. Si cumples con tu deber, Dios te lo premie y si no, te lo demande. Carlos Martí Bofill. ¡Viva Cristo Rey! ¡Viva España!»

El nacionalcatolicisme es consolidarà a Espanya. La doctrina de l'Església catòlica i les doctrines del Movimiento Nacional seran els eixos fonamentals de l'educació franquista. Ordre, disciplina, por, resignació, autoritarisme, etc. seran les bases de l'escola franquista. Mentrestant, els exiliats, lluny de casa seva, continuaran educant en la llibertat i en la responsabilitat. Mentre en aquelles escoles educaven per fer persones, fer ciutadans, a l'escola de la dictadura s'educava per fer súbdits.

\section{Notes}

${ }^{1}$ Preàmbul del decret de creació de l'Escola Normal, 22 d'agost de 1931.

2 Pròleg del decret de creació del Consell de l'Escola Nova Unificada, Barcelona, 27 de juliol de 1936.

${ }^{3}$ Després de la inauguració amb Mariano Rajoy de la línia d'alta tensió (MAT) entre França i Espanya va anar a la platja d'Argelers (Rajoy no el va acompanyar) a fer un homenatge als refugiats espanyols. Davant del monòlit de la platja va dir: «Sóc fill d'espanyols, d'una família republicana catalana.» I parlant dels refugiats, va afirmar: «Tots ells fugien de les tropes franquistes després de la caiguda de Barcelona i el que van trobar en arribar aquí van ser els camps del menyspreu. França no va estar a l'altura de les esperances d'aquella gent necessitada.»

${ }^{4}$ Aquesta és l'expressió oficial que utilitzaren les autoritats franceses: camps de concentració. Amb el pas del temps, i especialment després de la Segona Guerra Mundial, es va parlar de camps d'acolliment, camps de refugiats, etc.

5 J.J. Cruz (1994b, p. 7) apunta la necessitat de creixement de la població blanca per equilibrar la natalitat dels dominicans de raça negra. 
244 Marquès - 1939: L'escola renovadora de Catalunya

6 En el moment d'escriure aquest article, la filla del matrimoni m'acaba d'enviar unes memòries manuscrites inèdites de la seva mare, la mestra Munarriz, explicant la seva vida, primer de soltera i, en una segona part, la del matrimoni.

\section{Referències}

Cruz, J. I. (1994a). Los barracones de la cultura. Noticias sobre las actividades educativas de los exiliados españoles en los campos de refugiados. Spagna Contemporanea, 5, 61-78.

Cruz, J. I. (1994b). La educación republicana en América (1939-1992).

València: Generalitat Valenciana

Cruz Orozco, J. I. (2004). Maestros y colegios en el exilio de 1939.

València: Institució Alfons el Magnànim.

D. A. (2000). Una esperança desfeta. L'exili del 1939. Barcelona: Museu d'Història de Catalunya.

Fontquerni, E., i Ribalta, M. (1982). L'ensenyament a Catalunya durant la guerra. El CENU. Barcelona: Barcanova.

González Agàpito, J., i Marquès, S. (1996). La repressió del professorat a Catalunya sota el franquisme (1939-1943) segons les dades del Ministeri d'Educació Nacional. Barcelona: Institut d'Estudis Catalans.

González-Agàpito, J., Marquès. S., Mayordomo, A., i Sureda, B. (2002)

Tradició i renovació pedagògica. 1898-1939. Barcelona: Publicacions de l'Abadia de Montserrat.

Lozano, C. (1999). 1939. El exilio pedagógico. Barcelona: PPU.

Malgat, G. (2013). Gilberto Boques. La diplomacia al servicio de la libertad. París-Marsella (1939-1942). México: Conaculta.

Marquès, S. (1995). L'exili dels mestres 1939-1975. Girona: Universitat de Girona-Edicions del Segle.

Marquès. S. (2001). Riqueza para unos, pobreza para otros. Estudios Jaliscienses, Zapopan, 6, 24-37.

Marquès, S. (2002). Cultura republicana en el exilio francés. La cultura en los campos de concentración (1939-1943). A Cardillach, L., Peregrina, A. (coord.), Ensayos en homenaje a José María Murià. Zapopan: El Colegio de Jalisco. 
Marquès, S. (2003). Maestros catalanes del exilio. Zapopan, Jalisco, México: El Colegio de Jalisco.

Marquès, S. (2004). Los hermanos Bargés Barba, maestros renovadores en Cataluña y México. Zapopan, Jalisco, México: El Colegio de Jalisco.

Marquès, S. (2011). Educación republicana en Cataluña y Torreón. Jalisco, México: Instituto Nacional de Antropología e Historia.

Marquès, S., i Martín Frechilla, J. J. (2002). La labor educativa de los exiliados españoles en Venezuela. Caracas: Fondo Editorial de Humanidades - Universidad Central de Venezuela.

Pla, D. (1985). Los niños de Morelia. Un estudio sobre los primeros refugiados españoles en México. México: INAH.

Reyes, J. J. (1982). Escuelas, maestros y pedagogos. A D. A. El exilio español en México. 1939-1982. México: Salvat-Fondo de Cultura Económica.

Soler Mata, J. (2015). Vint mestres i pedagogues catalanes del segle XX. (Coord.). Barcelona: Rosa Sensat.

Dr. Salomó Marquès: professor al Departament de Pedagogia de la Universitat de Girona.

Contact Address: salomo.marques@udg.edu 


\section{HSE}

Historia Social y de la Educación

Social and Education History
Hipatia Press

www.hipatiapress.com

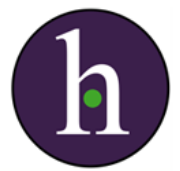

Instructions for authors, subscriptions and further details:

http://hse.hipatiapress.com

\section{Affirmative actions for minorities in India: Constrains and possibilities}

Mohd Sanjeer Alam ${ }^{1}$

1) Centre for the Study of Developing Societies (CSDS), India

Date of publication: October $23^{\text {rd }}, 2016$

Edition period: October 2016-February 2017

To cite this article: Alam, M.S. (2016). Affirmativa actions for minorities in India: Constrains and possibilities. Social and Education History 5(3), 246272. doi:10.17583/hse.2016.2245

To link this article: http://dx.doi.org/10.17583/hse.2016.2245

PLEASE SCROLL DOWN FOR ARTICLE

The terms and conditions of use are related to the Open Journal System and to Creative Commons Attribution License (CC-BY). 


\title{
Affirmative Actions for Minorities in India: Constrains and Possibilities
}

\author{
Mohd Sanjeer Alam \\ Centre for the Study of Developing \\ Societies (India)
}

\section{Abstract}

India is one of the most socially fragmented and unequal societies of the world. At the same time, it has the distinction of having the longest history of most elaborative affirmative action programmes for alleviating socially structured inequalities. While the affirmative action programmes have wider coverage in terms of social groups, there is continuing demand by new social groups for getting acknowledged as 'disadvantaged' and inclusion in the system of affirmative action. While group based 'reservation' as the most vital instrument of social justice has long been under fire and grappling with several challenges, the social justice regime is faced with the charge that it has largely excluded nation's religious minorities. Of course, religion based affirmative action is faced with many constraints; nevertheless there are possibilities for it. This article discusses the constraints and possibilities of affirmative action for disadvantaged religious minorities, Muslims in particular.

Keywords: Affirmative action, Disadvantage, Minority, Muslims, Equal Opportunity, Reservation, Social backwardness 


\section{Acciones Afirmativas para Minorías en la India: Limitaciones y Posibilidades}

Mohd Sanjeer Alam

Centre for the Study of Developing

Societies (India)

\section{Resumen}

India es una de las sociedades más fragmentadas y desiguales del mundo. Al mismo tiempo, cuenta con la particularidad de presentar la trayectoria más larga en la elaboración de programas de acción afirmativa orientados a hacer frente a las desigualdades estructurales. Mientras que los programas de acción afirmativa tienen una amplia cobertura en relación a los grupos sociales, existe una demanda constante por parte de nuevos grupos para conseguir el reconocimiento como "desfavorecido" y su inclusión en el sistema de acción afirmativa. Mientras que el sistema de cuotas como instrumento para promover la justicia social ha estado en el punto de mira y ha tenido que lidiar con diversos desafíos, las políticas de justicia social se enfrentan a la acusación de que se ha excluido en gran medida a las minorías religiosas del país. Por supuesto, la acción afirmativa basada en la religión se enfrenta a muchos obstáculos; sin embargo, existen posibilidades para ello. Este artículo analiza las limitaciones y posibilidades de la acción afirmativa para minorías religiosas desfavorecidas, especialmente en el caso de las personas musulmanas.

Palabras clave: acción afirmativa, desigualdad, minoría, personas musulmanas, igualdad de oportuniddes, cuotas, rezago social. 


\section{Alam-Affirmative Actions for Minorities in India}

T ndia is one of the most socially fragmented and unequal societies of the world. At the same time, it has also had the distinction of having (the most elaborated and longest running affirmative action programmes for alleviating structured inequalities (Galanter, 1984). ${ }^{1}$ In order to achieve greater socio-economic equality for historically disadvantaged social groups such as the Scheduled Castes (SCs) and the Scheduled Tribes (STs), there are fixed quotas for them (proportionate to their share in overall population) in educational institutions, public employment and political institutions (Parliament, State Legislative Assemblies and Local Bodies). In recent years, the system of reservation or quota has also been extended to Other Backward Classes (OBCs) whereby seats are reserved for them in public employment and institutions of higher education. However, there is continuing demand for reservation by new social groups. In many cases, the social groups struggling for reservation happen to be relatively prosperous and locally powerful. ${ }^{2}$ The continuing demand for and dispute over getting acknowledged as 'disadvantaged' have raised many questions with regard to conceptualization of disadvantage and the rationale, form, capacity and implications of the existing affirmative action programmes. While group based 'reservation' as the most vital instrument of social justice has long been under fire and grappling with several challenges, the social justice regime is faced with the charge that it has largely excluded nation's minorities.

As per the constitutional provisions religion based affirmative action is proscribed, although some religious minorities, if not all, have long been projecting them as disadvantaged and excluded, and, therefore, demanding for their inclusion in the affirmative action programmes. Nevertheless, the issue got renewed vigour following the findings and recommendations of the two commissions appointed by the Government of India - (a) The National Commission for Religious and Linguistic Minorities (NCRLM) constituted in 2004 under the chairmanship of Justice Ranganth Misra; and (b) Prime Minister's High Level Committee to prepare 'A Report on the Social, Economic and Educational Status of Muslim Community of India' set up in 2005 under the chairmanship of Justice Rajinder Sachar (henceforward Sachar Committee Report). While the former recommended for reservation 
for religious minorities as in the case SCs/STs/OBCs, the latter recommended for a range of affirmative measures (other than reservation) for disadvantaged religious minorities, Muslim minorities in particular.

But the idea of affirmative action for Muslims or for that matter any other religious group as a 'disadvantaged group' is beset with many fundamental challenges. These include but are not limited to: (a) legal/constitutional barriers to religion based affirmative action ${ }^{3}$; (b) complex intra - group differentiation and inequality; (c) finding a convincing way of measuring disadvantage in its all complexity; and (d) minimizing the impact of unintended policy consequences (social and political turmoil). Nevertheless, given the pathetic average socioeconomic conditions of the community, there is a compelling need for state intervention so as to enable them to have greater access to and participation in those opportunities availed by majority of people (Alam, 2010; 2014).

Against this backdrop the aim of this paper is threefold: (a) to understand the sociopolitical processes underlying affirmative action for religious minorities; (b) to interrogate various strands of debate on affirmative action for Muslims, and (b) to propose and critically examine various policy alternatives in order to enrich the ongoing debates and churning on affirmative action for Muslims.

The remainder of the paper is structured as follows. The following section briefly discusses as to constitutional position on minorities and how they are educationally and economically placed. Section 3 presents the contours of affirmative action debate on religious minorities. Section 4 discusses various models through which Muslim minorities may be benefited from existing affirmative action programmes. Section 5 concludes the discussion.

\section{India's National Minorities}

In India, the word 'minority' is quite loosely defined and continues to be debated. The Constitution speaks of religious and linguistic minorities. Article 30 of the Constitution speaks specifically about two categories of minorities - religious and linguistic. Religious communities, which are culturally distinct and numerically smaller than the Hindus at national level, are designated as a 'minority'. Seen thus, religious groups such as Muslims, 


\section{Alam - Affirmative Actions for Minorities in India}

Christians, Sikhs, Jains, Buddhists and Parsis are recognized as national minorities. $^{4}$

Although there is a legal/constitutional framework for protection of minority rights, some minority groups, if not all, are found to face severe socioeconomic disadvantages for various reasons. For example, religious minorities such as Parsis, Christians, Sikhs and Jains are much better on a variety of indicators of human development than the national average or Hindus (the majority community) taken as a whole. Thus, minority status per se is not linked to socioeconomic disadvantage and deprivation. However, the Muslims, the largest religious minority group (constituting about 14 percent of total population), have fallen behind others in the crucial sectors of life (Sachar, 2006; Alam, 2009a, 2009b, 2010; Shariff \& Basant, 2010). And, therefore, the debate on affirmative action for minorities in the current context is largely confined to the Muslim community.

\section{Debating Affirmative Action for Minorities}

During the colonial rule, religious minorities enjoyed benefits of affirmative action in various spheres (Wright, 1997). After the colonial power gone, these privileges given to minorities were done away with. The Constitution of India did not provide for affirmative measures for religious minorities as it did for formerly 'depressed classes'. Nonetheless, the issue was intensely debated in the Constituent Assembly. In the Constituent Assembly Debates (CAD) meetings held between December 1946 and August 1947 reservation was seen as a possible solution to allay fears and apprehensions of minorities (Hasan, 2009; Tejani, 2013). For instance, the sub-committee on minority rights in its report (submitted to the Advisory Committee on Fundamental Rights on the $27^{\text {th }}$ July 1947) had recommended for reservation for religious minorities such as Muslims, Christians and Sikhs in legislatures on the basis of joint electorates and in public employment in proportion to their share in overall population. ${ }^{5}$ Accordingly, the Advisory Committee on Fundamental Rights recommended that seats for different recognized minorities as a rule would be reserved. However, the issue reopened following the report of a special sub-committee that had met in December 1948. ${ }^{6}$ The report held that the situation in the country (following the Partition) had changed to such an extent that 'it was no longer appropriate in the context of Free India and of 
present conditions that there should be reservation of seats for Muslims, Christians and Sikhs or any other religious community'. It further held that reservation for religious communities 'did lead to a certain degree of separatism'. 7 As a matter of fact, majority of members belonging to minorities held that political safeguards would not serve the interest for minorities. Thus, acting upon the report of the special sub-committee, the Advisory Committee overwhelmingly voted for the abolition of political safeguards for religious minorities.

The reservation for religious minorities in services reopened for discussion in the CA on the $14^{\text {th }}$ October 1949. Moving amendment (No. 3163 of the list of amendments) for Article $296,{ }^{8}$ B.R Ambedkar proposed that ' $\mathrm{t}]$ he claims of members of the Scheduled Castes and Scheduled Tribes shall be taken into consideration consistently with the maintenance of efficiency of administration, in the making of appointments to services and posts in connection with the affairs of the Union or of a State'. ${ }^{9}$ Many members including Sardar Hukum Singh, Bhopindra Singh Mann and Naziruddin Ahmad (all belonging to religious minority communities) pointed out that the present resolution was reversal of earlier decision. Sardar Hukum Singh argued that it was nowhere suggested that all safeguards would go. The only decision that was agreed to was: "That the system of reservation for minorities other than Scheduled Castes in legislatures be abolished". Naziruddin Ahmed submitted:

"It is absolutely clear on a perusal of the original report, the letter of Sardar Patel, the resolution moved by him and in the speeches in the Houses - that they all attempted reconsideration only of the reservation for minorities in the Legislatures. I may add that this was done with the fullest concurrence of the Muslim members of this House. I was one of those who thought that the reservation in Legislatures would not be good for minorities themselves; but with regard to consideration of their cases in making appointments, subject to efficiency was not reopened' ${ }^{10}$

Sardar Hukum Singh made a powerful plea for restoring the original proposal that was already accepted by the House. He proposed that article 296 be substituted to read: "Subject to the provision of the next succeeding 


\section{Alam-Affirmative Actions for Minorities in India}

article the claims of all minority communities shall be taken into consideration, consistently with the maintenance of administration, in the making appointments to services and posts in connection with the affairs of the Union or of a State for the time being specified in Part I of the First Schedule." When put to vote, his amendment was rejected by the House and that of Ambedkar voted to become part of the constitution. With this, reservation for minorities was wholly rolled back.

What explains change of mind of the members of constituent assembly? It is suggested that it was largely the partition and consequent communal violence that served a major blow on the scope of reservation for religious minorities. First, the Partition marked departure of 55 Muslims, leaving behind only 28 members in the constituent assembly. With this, the bargaining power of the members of the minority communities in the constituent assembly got diluted substantially. Secondly, the changed circumstances forced the members of minority communities to demonstrate their loyalty to India. As Wright Jr. (1997, p. 853) puts it: 'neither were Hindu representatives willing to continue this concession nor were the remaining Muslim committee members prepared to press for it'. Now on, the minority rights got increasingly framed under "secularism" and "undifferentiated citizenship" rather than "social justice". Many members of constituent assembly argued that in secular India political safeguard along religious lines would be antithetical to the ideals of secularism. For example, Krishnaswamy Bharti from Madras argued that "community should not be made the basis of civic right. In a secular State right to representation is only the right to represent a territory in which all communities live". ${ }^{11}$ Other members namely Mahabir Tyagi also made similar points. In the given political circumstances, not only did these voices have wider appeal in the assembly, they were difficult to be countered.

A fresh opportunity for minorities to get included into the reservation system arose when the first Backward Classes Commission was set up in 1953 to identify 'backward classes' and widen the coverage of affirmative progrommes. The commission received representations from religious organizations. In their representations, Muslim organizations demanded that the Muslim community as a whole should be treated as backward. While the commission did not treat religious communities (minority religions) as a 
whole as a backward class, it recognized some groups/sub-groups among them as 'backward classes'. ${ }^{12}$ Like its predecessor, the second Backward Classes Commission constituted in 1979 (popularly known as Mandal Commission after its chairman B.P Mandal) also did not recognize religious groups as a whole as 'backward class'. Rather, it identified some groups among non-Hindus as socially and educationally backward classes based on the two criteria: (a) all untouchables converted to non-Hindu religions; and (b) such occupational communities which are known by the name of their traditional hereditary occupation and whose Hindu counterparts have been included in the list Hindu OBCs. Though the criteria for identifying OBCs among non-Hindus were short of careful thought and beset with methodological fallacies, the fact remains that a section of Muslims became part of the reservation system.

The inclusion of some groups/sub-groups, however, did not satisfy the community elites. The demands for reservation for the entire community continued. In 1994, the Association for Promoting Education and Employment of Muslims (APEEM) convened a conference in New Delhi. This conference was of immense importance because the then Minister of Welfare in the Union Cabinet, Sitaram Keshri, not only attended the conference but also advocated separate quota for Muslims in educational institutions and public employment. This implied that the Conference had been convened with the tacit support of the Congress (Wright, 1997). The APEEM has continued to buttress demands of reservation for Muslims in its successive conferences (Alam, 2009a, 2009b).

The idea of affirmative action for Muslims received wider attention following the reports of the two commissions set up by the UPA (United Progressive Alliance) government in 2004. The Prime minister's High Power Panel, popularly known as Sachar Committee, analyzed socioeconomic status of the Muslim community vis-à-vis others. ${ }^{13}$ Based on its findings on certain indicators of educational and economic attainment social groups can be vertically arranged as follows: forward caste Hindus at the top, followed by Hindu OBCs as well as upper caste/class Muslims; distantly followed by SCs/STs, and the Muslim OBCs being at the bottom. While the primary mandate of the Sachar Committee was to map the levels of deprivation among Muslims and diagnose the problem, it also highlighted 


\section{Alam - Affirmative Actions for Minorities in India}

the areas of intervention and suggested multifarious measures (minus reservation) to overcome various kinds of deprivations among Muslims.

The 'National Commission on Religious and Linguistic Minorities' (NCRLM) appointed around the same time was, however, primarily mandated to find out solutions to socioeconomic problems facing the Muslim community. Its mandate included: (a) suggest criteria for identification of socially and economically backward sections among religious and linguistic minorities; (b) recommend measures for welfare of socially and economically backward sections among religious and linguistic minorities, including reservation in education and government employment; and (c) to suggest the necessary constitutional, legal and administrative modalities required for the implementation of its recommendations. Accordingly, the NCRLM in its report (2007) recommended reservation for Muslims. We will discuss its proposals with regard to reservation for minorities in greater detail in the following section; suffice here to say that it gave some legitimacy to the demands of reservation for Muslims.

\section{Alternative Models of Affirmative Action for Minorities: Constraints and Possibilities}

In India, much of the discourse on affirmative action is focussed on reservation. Nevertheless, there are many important ways through which group based disadvantages can be substantively redressed. In fact, many of them appear to be more capable of combating group based disadvantages than reservation. In what follows is a critical discussion on a wide array of approaches or models that might contribute to or could be useful in thinking about social equity for disadvantaged minorities such as Muslims.

\section{Quota Approach}

It is probably the most popular as well as contentious form of affirmative action. The popularity of quota or reservation lies in the fact that it is an outcome oriented policy approach, for it involves fixed amount of budgetary allocation and/or a fixed proportion of seats in educational institutions and 
public employment reserved for the disadvantaged group. This form of affirmative action is particularly effective in the societies that are deeply divided along religious, ethnic or linguistic axes and characterized by deep institutional and structural barriers to social equality. Since a quota for the disadvantaged group in question is "fixed", it helps disadvantaged groups overcome prejudices against them in the system of distributive benefits and the wider society as well.

The most distinctive aspect of affirmative action in India is fixed quota for the target groups - the Scheduled Castes (SCs), the Scheduled Tribes (STs) and the Other Backward Classes (OBCs). As far as inclusion of Muslims in the reservation policy is concerned, some sub-groups among them are identified as OBCs. However, it is often claimed that Muslims have not received a fair deal in the reservation policy. While there is a demand for a fixed quota for the entire Muslim community, it has several strands. There are many who argue that relative backwardness of Muslims is more a matter of inter-group diversity than intra-group disparity, for Muslims as a whole are victim of invidious discrimination that has pushed them to the level of SCs/STs. (Akhtar \& Ahmad 2003; Hasan, 2005). The nub of these arguments is that the identity based biases run so deep in the system that not only are affirmative actions necessary, but no affirmative action policy other than 'fixed quota' is ever likely to work.

Such arguments have, however, come under attack from within the community itself. Some sections of Muslims argue that class and caste categories should be retained while considering Muslims for affirmative action (Engineer, 2004). It is argued that like Hindus, Muslims are also characterised by internal differentiations in terms of caste-like groupings. Though Islam prohibits caste and class distinctions among Muslims, in practice the Muslim community is not immune from caste-like hierarchies (Ahmad, 1978; Karanath, 2007). More recent converts, the majority of whom were lower caste Hindus, are not seen as socio-economic equals within the community (Ali, 2001; Mondal, 2003). This implies that all Muslims are not backward, nor do all of them require affirmative action. The basis of affirmative action in the form of reservation for Muslims should, therefore, be linked to social and economic stratification within the community. The underlying assumption is that the benefits of reservation 


\section{Alam - Affirmative Actions for Minorities in India}

should go to those within the Muslim community whose social and economic status is no different from Hindu lower castes. Extending benefits of reservation to the entire community would only benefit the upper crust of the community who can advance in life on their own, while those who really need reservations would be left untouched. Hence, it will defeat the avowed purpose of the policy aimed at uplifting the deprived sections within the community. This argument is particularly advanced by the Pasmanda (backward) Muslims Organizations (Jenkins, 2003).

The official proposal for extending reservation to the entire Muslim community is put forward by the NCLRM (2007). There are basically two proposals. One, the entire Muslim community may be declared 'backward' within the meaning of that term in Article 16 (4) of the Constitution notably without qualifying the word 'backward' with the words 'socially' and 'educationally' and that reservation could be given over and above existing 49.5 percent. The commission suggested 15 percent reservation for minorities, subdivided into 10 percent for Muslims and 5 percent for other minorities. The second proposal is, in fact, placed as an alternative in case there is any difficulty in implementing the first proposal. According to this proposal, subject to minor adjustment inter se in accordance with population of various minorities in various states/UTs, a sub quota of 8.4 for minorities (internally sub-divided into 6 percent for Muslims and 2.4 percent for other minorities) within the 27 percent $\mathrm{OBC}$ quota should be carved out on the ground that minorities account for 8.4 percent of the total OBC population. ${ }^{14}$ However, both the proposals appear to be faced with legal/constitutional problems. As far as the first proposal is concerned, recognising the entire Muslim community as 'backward' for the purpose of reservation involves amendments in the constitution for (a) extending reservation to Muslims as a backward class' and (b) removing the ceiling of 50 percent. ${ }^{15}$ On the second proposal, it has been argued that the inclusion of groups in the category of OBC is based on the twin criteria of 'social' and 'educational' backwardness. As stated earlier, since Indian Muslims comprise of multitude of sub-groups and many of them are of high social origin, the idea of inclusion of the entire Muslim community is not in the fitness of constitutional provisions. 
One may, of course, argue that if objective position of Muslims as a whole is no different from officially recognized backward classes, the constitution should be amended to recognize them as 'backward classes. After all, the constitution has been amended for similar purposes on many occasions. For example, it has been amended to grant the status of the Scheduled Caste to members of Sikh and Buddhist communities in 1956 and 1990 respectively. On the face of it, this argument has some merits. However, arguments such as these should not be made in isolation from larger social and political consequences. For one, if past experience is any guide, reservation has been an overly emotive and divisive issue. Attempts of increasing the quantum of reservation have met with violent resistance and political backlash (Kumar, 1992; Shah, 1991; Weiner, 1983). The extension of reservation to Muslims would not only be opposed at political and societal levels, ${ }^{16}$ the reactions would be even more violent than in the case of OBCs. In other words, pressing for reservation for the entire Muslim community in the current socio-political clime may further isolate the community from the wider society. Secondly, as we have noted, the opposition to the idea of reservation for the entire Muslim community is also likely to come from within the community itself. In other words, considering all Muslims for reservation may also create many problems within the community itself.

In view of above complexities a pertinent question that arises: is there any scope under the existing legal/constitutional framework and political conditions to maximize the benefits of reservation for eligible Muslims? There are two issues to be looked into in this regard. It is often claimed (a) that the method used by the Mandal Commission to identify OBCs among Muslims is flawed, leaving many genuinely deserving sub-groups among Muslims out of the OBC category; (b) that Muslim OBCs are far more disadvantaged than their counterpart among Hindus and as a result the former are unable to compete with the latter and thus much less likely to benefit from reservation earmarked for OBCs as a whole.

Let us take up the first claim first. As per the NSSO (68 ${ }^{\text {th }}$ round) estimates, Muslim OBCs account for 7 percent of total population. In other words, half the Muslims (13.4 percent of total population) are placed in the OBC category. However, sociologists and anthropologists have suggested 


\section{Alam-Affirmative Actions for Minorities in India}

that about 80-90 percent of Muslims are descendents of converts who belonged to 'untouchables' and lower caste Hindus (Mondal, 2003). It thus points to the fact that the Muslim communities who are socially and educationally backward like their counterparts following other religions have not been properly identified. To put slightly differently, there is scope for identifying many more Muslim communities as socially and educationally backward classes and place them in the OBC list. Of late, this exercise has been done in a number of states including West Bengal and Andhra Pradesh. It has not faced hurdles of any sort - legal or political. In brief, identification and inclusion of more Muslim communities in both central and state lists of $\mathrm{OBCs}$ is possible without any fetters. If identification problem is overcome it will substantially increase Muslims' share in the reservation policy.

With regard to the claim of not being able to appropriate benefits of reservation, it is not the Muslim OBCs who are particularly faced with this problem. As a matter of fact, a large number of communities have complained that they are unable to compete with relatively advanced communities within the OBCs, and therefore not benefiting from the reservation regime. Acting upon such complaints, many states have created sub-quotas within the 27 per cent quota. This also includes a sub-quota for OBC Muslims. For example, Kerala has divided OBC into eight categories and accordingly has eight sub-quotas. There is one sub-quota for Muslim OBCs. Similarly, Karnataka has a sub-quota of 4 per cent for Muslim OBCs. Recently, Andhra Pradesh has added the category ' $E$ ' in the existing fourfold classification of OBCs to devise a sub-quota of 4 per cent for Muslim OBCs (Krishnan 2012). Such classifications and sub-quotas have stood judicial scrutiny. Nor have they been subjected to political controversies. In brief, sub-quotas for most backward communities including Muslims (OBCs) are legally appropriate, judicially sustainable and politically non- contentious. Thus, if a comprehensive system for sub-quotas based on differential levels of backwardness/deprivation at the central and state level (where sub-quotas are not available) is done, it will benefit the most backward communities including Muslims identified as OBCs. 


\section{Spatial Approach}

The spatial approach is rather a process oriented policy. It is most suited in the context where ethnicity based deprivation is coterminous with backwardness of region. Nigeria offers the best example of this model of affirmative action. This approach envisages undertaking of various ameliorative schemes for the development of the backward region, leading to betterment of the local communities inhabiting the given region. A distinctive feature of this approach is that spatiality masks both ethnicity and class as the basis of selection of beneficiaries, a major critique of the 'quota' approach. As the coverage of this form of affirmative action is broad based, programmes undertaken are socially and politically least contentious. However, such an approach may not yield desired or desirable results for various reasons. For one, disadvantaged groups are often poor in human and social capital. In such events, developmental initiatives may bypass the intended beneficiaries even in areas where they are in greater numbers. Second, biased bureaucracy or policy implementing agencies may distribute funds or tailor schemes in such a manner that ends up benefiting the well off groups/individuals.

How far does this approach suit affirmative action for Muslim minorities? In order to be able to answer this question, we need first of all to look at spatial demography of Muslims. In 2001, Muslims with a population of 138 millions accounted for about 13.4 percent of total population in the country (Census of India 2001). Unlike other minority communities, Muslims are unevenly distributed across the length and breadth of the country. At the national level, the proportion of Muslims varies from 66.9 percent in Jammu and Kashmir and 30.9 percent in Assam to 5.5 percent in Tamil Nadu. Of the total Muslim population in the country, over half (53 percent) lives in just four states namely Assam, Bihar, Uttar Pradesh and West Bengal. The southern four states - Andhra Pradesh, Karnataka, Kerala and Tamil Nadu - together account for one-sixth of total Muslim population in the country. At the sub-national level, while there is hardly a district that does not have Muslim population, there are 20 districts (out of 594 districts in 2001) across states where Muslims form the majority community. There are another 38 districts that have substantial Muslim population, accounting 


\section{Alam - Affirmative Actions for Minorities in India}

for over a quarter of total population but below 50 percent. The spatial distribution of Muslims does suggest that there are some clusters of Muslims and therefore spatial approach may be applicable as well as useful in designing affirmative action for Muslims. Let us now take a brief look at different aspects of spatial approach including coverage, efficacy and quantum of benefits.

In India, the spatial approach in the context of religious minorities has been in existence since 1987. Partly as a follow up to the Gopal Singh Committee Report, the Government of India prepared a list of 41 minority concentration districts (having 20 per cent or more population of minorities in a district based on 1971 census) for focused attention of the government to implement developmental and welfare schemes. In 2008-09, the Government of India revamped this approach to launch area development initiatives, also known as 'multi-sectoral development programme' (MsDP). This programme was launched in 90 minority concentrated districts (MCD) (based on 25 percent or more minority population as per 2001 census). However, the MsDP progrommes came under attack for several reasons such as inadequate coverage, flawed design and poor implementation. .

In terms of coverage, MCDs contained only 37 percent of minority population. As far as Muslims are concerned, they accounted for 30 percent of total population in the MCDs and 40 percent of total Muslim population the country. Thus, majority of Muslims lived outside the MCDs, and therefore left out of the MsDP programme. The MsDP also had serious design, content and implementation problems. It was designed as an umbrella programme to intensify developmental activities in the MCDs. Since the district was made unit of planning, funding and implementation in absence of clear guidance as to where in the districts the projects were to be carried out, the schemes ended up benefiting non-minority population. On content, the schemes launched under MsDP were not only very few but also did not promise far reaching changes in the lives of minorities. For instance, these schemes basically related to housing (Indira Awas Yojna), construction of primary schools, more specifically construction of additional classrooms, skill development initiatives etc. Again, in absence of clear guidelines on selection of activities, bulk of spending was reported to have been directed towards construction of houses (Khan \& Parvati, 2013). 
Realizing that district is too big a unit for planning, the government has now switched to sub-district as a unit of planning, funding and implementation of projects. A total of 710 minority concentration blocks (with 25 percent or more minority population) in 155 backward districts have been identified. Moreover, in selected sub-districts (blocks), as the new guidelines suggest, the villages having higher minority population would be given priority for creation of village level infrastructure/assets. With this, part of the coverage problem seems to have been addressed. Over 60 percent of Muslim population is now covered by minority concentrated blocks (MCBs).

However, the problems with regard to content and implementation remain. To make this approach contributing significantly to address developmental deficits in minority concentrated areas, there is a need to enlarge the bouquet of schemes and remove the institutional bottlenecks. On the front of education, for example, the focus should shift from construction of primary and upper primary schools to construction of secondary schools, colleges and technical institutes (other than ITIs). In each of the identified block schools matching the quality of Kendriya or Navodaya Vidyalay need to be opened up. On the economic front, funds exclusively for up-scaling home based enterprises may be made available on easy terms and conditions. There should also be focus on creating durable assets and sustainable employment opportunities. The list is too long to be presented here.

However, no amount of well intentioned schemes/programme can achieve anything unless they get implemented on the ground in true spirit of the schemes; and policies cannot get implemented unless the structures of implementation are simplified. Currently, the schemes launched under area initiative programme are jointly funded by the central and state governments. At a given point of time, different states have different parties in power which are differentially sensitive to the issues facing the minority communities and therefore policies aimed at benefiting minorities can be differentially successful across states. ${ }^{17}$ Moreover, there are multiple layers of planning and approval of activities. This offers ample scope to bureaucracy for delaying approval and implementing projects. In other 


\section{Alam-Affirmative Actions for Minorities in India}

words, this approach to affirmative action despite having huge potentials may prove a damp squib.

In sum, if carefully designed and effectively implemented, the spatial approach can greatly help address developmental deficits among Muslims along with others living in backward or under developed areas/regions.

\section{Sectoral Approach}

The sectoral approach involves identification of sectors for state intervention which may largely benefit the disadvantaged/targeted groups. Like spatial approach, the basis of selection of intended beneficiaries is not 'group' based on ascriptive characteristics, for its explicit aim is to bail out the entire sector identified for government intervention. And yet, this approach can be used to benefit individuals without making explicit reference to their ethnic or religious identity. An ideal context in which sectoral approach can be useful is the one where the disadvantaged group in question displays higher concentration in the given sectors of economy or occupations.

A major advantage of sectoral approach is that it is inclusive by design and thus not prone to legal and political controversies. For example, if a particular kind of enterprise is chosen for investment or for weeding out problems facing it, the entire sector gets benefitted. Although it is possible that a particular social group has disproportionate presence in that enterprise, it does not have exclusive claims on the benefits out of state intervention. Hence, such policies enjoy greater acceptance.

As far as application of this approach for addressing disadvantages faced by Muslims is concerned, it sounds great as a large number of Muslims are artisans and run home based enterprises. There are certain enterprises or occupation in which Muslim workers are disproportionately engaged. These enterprises include carpentry, butchery, weaving, lock and brass making, carpet and perfume making, leather work etc. Many of these enterprises face the problems of raw materials, financial capital, spatial segregation, lack of availability of appropriate modern technology and exploitation by big traders and so on. The onset of globalization and liberalization accompanied by unbridled powers of traders has further worsened the conditions of those employed in these occupations (Harris-White, 2003; Ahmad, 2013; Jamil, 2014). Thus, a policy package that provides for up-scaling and modernizing 
small scale enterprises by making capital available; up-skilling manpower (to use technology) by providing training; putting in place a mechanism to do away with exploitation by traders and so on may help a large number of Muslims and may have multiplier positive effects on their lives.

In India, sector based schemes have been in existence for some time. For instance, the Ministry of Cotton Textile has launched a number of schemes for handloom and handicraft sector. The handloom sector alone employs over 65 lakh persons. ${ }^{18}$ The government has identified about 470 handloom clusters for development of the handloom sector. In 2008-09, Comprehensive Handloom Sector Development Scheme was introduced by the Ministry of Cotton Textile for development of two Mega Handloom Clusters - Varansi (Uttar Pradesh) and Sivsagar (Assam). There are also schemes for other small scale enterprises launched by Ministry of Small Scale Industries.

On the face of it, sectoral approach may greatly help members of the Muslim community as the size of artisan class among them is disproportionately large. However, there are no studies to show how different schemes for traditional and marginal occupations work and impact the lives of those engaged in such occupations on the ground. Since most of such schemes are financed and implemented within the federal framework, that is, both central and state governments being responsible for financing, the efficacy of such schemes is likely to be variable across states depending on commitment of the state governments towards the welfare of people engaged in marginal occupations. Also, it is possible that the targeted groups may be used as the front but benefits of government schemes are actually cornered by others. In Malysia, this kind of arrangement is known as 'AliBaba' enterprises (Sowell, 2004). In this case, Ali is someone who belongs to the targeted groups (the native Bhumiputras) and in whose name the enterprise/firm is registered. But actual control of the firm/enterprise is in the hand of 'Baba' who is someone outside the targeted group.

\section{Anti- Discrimination Structures}

In multi-ethnic/multicultural societies, the minority groups based on ethnicity, region, religion, language, national origin etc., tend to have feelings of being discriminated against by the state agencies as well as the 


\section{Alam - Affirmative Actions for Minorities in India}

wider society. They often attribute their deprivations to invidious discrimination practised against them. In order to address the problem of discrimination - actual or perceived - many democratic and multi-ethnic countries - the United States of America, Canada, United Kingdom, Brazil, South Africa to name a few - have enacted anti-discrimination laws and created anti-discrimination or equal opportunity authorities. For example, the United States of America passed Equal Opportunity Act in 1972 and created Equal Employment Opportunity Commission to prohibit discrimination in employment and workplace. In 2010, the United Kingdom (UK) passed Equality Act 2010 to replace all previous anti-discrimination laws with a single Act. The Act aims to prohibit discrimination on the basis of race, religion, gender, sexual orientation, marital status and age. Without giving the whole list of countries with equal opportunity structures, suffice it to say that such measures are in existence and have proved useful to combat discrimination and problems of under- representation of disadvantaged groups in critical sectors of life such as employment and educational institutions. As Borooah (2010, p. 33) puts it, 'not only do EO policies attempt to eliminate discrimination bias by making it illegal, EO builds nondiscriminatory policies into the fabric of the human resource management of organizations...'. These efforts are said to have made substantial impact on the lives of the minority groups often prone to discrimination (Khaitan, 2008).

India is a multi-ethnic and multicultural country. As a matter of fact, almost all social groups, big or small, perceive themselves as potential victims of illegitimate discrimination. However, such feelings are more pervasive among historically marginalized groups and religious minorities. Whether or not a group is actually discriminated against, the feeling of discriminatory treatment itself causes a lot of damages. To illustrate, if a social group is gripped by the perception that its members are not going to get good jobs despite having appropriate qualification because of biased labour market, it may not invest in education and apply for jobs available in the labour market. In economic literature, this is called 'discouragement effect'. In brief, this traps the social group having perception of being discriminated against into the vicious cycle of lower investment in human capital (because education may not be seen as a passport to public 
employment), under-skilled manpower, lower level of economic mobility (because of lack of education), and lower level of income (because of being positioned at lower rungs of economic structures), and lower investment in human capital (because of lower income). It is thus absolutely necessary to create equal opportunity structures armed with statutory powers to deal with complaints of discrimination in a fixed time frame.

The first attempt towards creating Equal Opportunity Commission (EOC) was initiated by the UPA (United Progressive Alliance) government as a follow up to the recommendations of Sachar Committee (2005). The government appointed an Expert Group in 2008 to 'examine and determine the structure of an Equal Opportunity Commission'. Underlining the importance of Equal Opportunity Commission, the expert group observed: "Perhaps equal opportunity situations would have been different had the Government set up Equal Opportunity Commission immediately after the enactment of the Constitution". ${ }^{19}$

Given that EOCs in other countries have been very effective in weeding out discrimination in hiring processes and in distribution of state resources, this is not an exaggerated statement. The Commission envisaged an EOC that would be 'autonomous of the government of the day and be capable of responding quickly and effectively to any challenge it is faced with'; would entail multifarious functions - advisory/consultative, policy intervention, investigative and grievance redressal; would cover disadvantaged groups based on an open ended list of irrelevant personal characteristics; and would have jurisdiction over both public and private sectors. This makes the design of EOC not only morally better but also promises to positively affect the lives of disadvantaged groups/individuals far more than any other welfare measures can for the following reasons. First, since the principal reason for under-representation of disadvantaged groups including Muslims in various structures of opportunities is seen to lie in systemic discrimination against them, an effective EOC will not only help them redress their grievances sooner than later, it may instil confidence among them and thereby dilute the tendency (among disadvantaged groups/individuals) of withdrawing from competition for positions. Second, the jurisdiction of EOC is not just limited to jobs and educational institutions and that too in the public sector as in the case of reservation, but may also extend to unfair treatment in private sectors 


\section{Alam - Affirmative Actions for Minorities in India}

and a wide variety of other areas such as housing, institutional credit lending and so on. In other words, anti-discrimination measures including EOC can bring about far reaching changes in the lives of far more people across groups than reservation does or can.

Nevertheless, constitution of EOC like bodies is faced with two major, though not insurmountable, difficulties. One, there are many commissions dealing with issues of different groups with some functions similar to those proposed for EOC. In other words, there is scope of overlapping spheres of mandate and jurisdictions between EOC and other commissions or statutory bodies like Commissions for Scheduled Castes and Scheduled Tribes, Women's Commissions, Minority Commissions and so on. One way out of this impasse is to merge different bodies/structures/commissions into a single Equal Opportunity Commission or to take out some powers/functions (relevant to EOC) of different commissions and assign them to EOC. But this is not an easy task to do. This is precisely the reason why the EOC Bill could not be introduced in the Parliament. There were differences of opinions within the government. One argument was that since EOC as proposed by the Expert Group would stand in conflict with other existing statutory commissions, it should cover religious minorities only. Others argued that EOC for minorities only would render it meaningless. It will be seen as another instrument of minority appeasement. Second, even an effectively functioning EOC may end up dealing with limited number of complaints. Given the pervasiveness of discriminatory feelings across social groups, the EOC will be flooded with the complaints of discrimination which in turn may seriously impair its ability to dispose of the cases in the given time frame. In such a situation, to have an EOC may mean like any other commissions that hardly serve the purpose for which they were created.

In sum, given the pervasiveness of discrimination the EOC can be an effective instrument of addressing social inequities, provided that it is designed in much better ways than the one proposed by the government, that is, for minorities only. 


\section{Conclusion}

India is one of most unequal societies of the world. Inequalities are manifested in terms of caste, tribe, gender, religion and region and so on. These axes of inequalities are, however, not mutually exclusive. They tend to get nested in complex ways. Nonetheless, religion is a major axis of persistent inequalities. Given that the Muslim communities are faced with several forms of disadvantages relative to other socio-religious communities, and that India has the most elaborated affirmative action programmes for disadvantaged groups, there is a strong case of affirmative action for Muslims. However, designing affirmative action for Muslims or for that matter any other disadvantaged minority group should be based on sound reason and criteria to convey right messages to the beneficiary group as well as the wider society. So, affirmative action policy for Muslims needs to factor in the followings: (a) that the nature and degrees of disadvantages facing Muslims vary across space and also in terms of social origin, that is, their relative backwardness is multi-layered; (b) that the existing constitutional provisions put some limitations to adoption of affirmative measures for Muslims similar to other disadvantage groups; and (c) the political class is sharply divided on different forms of affirmative action, if not on the idea of 'affirmative action' for minorities per se. It thus suggests that a single set of measures will be inadequate to remove complex and multi-layered disadvantages facing the Muslims. To put slightly differently, there is a need is to follow what may be called a "mixed approach" or a "multilateral approach". For example, at one level initiatives may be taken to proper identification of backward classes among Muslims. At another level, spatial and sectoral approaches either combined or separately can be better designed and implemented to benefit Muslims along with other deprived groups to tackle developmental deficits thereof. At the other level, antidiscrimination measures such as EOC may be put in place. Taken together, these measures can be reasonably expected not only to help addressing grievances of Muslims and other marginalized groups but also to bring about substantive transformation in their lives. 


\section{Alam - Affirmative Actions for Minorities in India}

\section{Notes}

${ }^{1}$ Affirmative action is a nebulous term used to refer to deliberate undertaking of positive steps or policies aimed to make progress towards achieving substantive rather than formal equality of opportunity for those groups currently underrepresented in crucial sectors of life and/or in significant positions in the structures of opportunities (Mullen 1988; Brest and Oshige, 1995). In India, policies that can be described as affirmative action were introduced by the British regime in the very beginning of the $20^{\text {th }}$ century for religious minorities and depressed classes. In the first quarter of the $20^{\text {th }}$ century, middle castes were given benefits of reservation in public employment in the Madras Presidency and some of the independently ruled areas of southern and western India.

${ }^{2}$ For instance, the Jats, the Marathas and the Patels are land owning and relatively prosperous communities and yet demanding for reservation. Out of political expediency, the state governments carved out quotas for them but such quotas could not stand judicial scrutiny and were summarily struck down by the courts. On the other hand, the Gujjars, traditionally a pastoral community of north and central India, have been agitating for some time to be included in the list of STs. They claim that they are tribes and have wrongly been placed in the list of OBCs.

${ }^{3}$ For example, the Andhra Pradesh Government introduced a quota of 5 per cent for Muslims in educational institutions as well as public employment for Muslims. It was challenged in the court and eventually the Andhra Pradesh High court struck it down.

${ }^{4}$ Also see National Commission for Minorities Act 1992; Report of the National Commission for Religious and Linguistic Minorities, Ministry of Minority Affairs, Government of India, 2007.

5 Also see CAD (26 May 1949:321).

6 The members of the sub-committee comprised J.L Nehru, Rajendra Prasad, K.M Munshi and B.R Ambedkar and Sardar Patel. Sardar Patel, on 11 May 1949, laid down the report of this sub-committee before the Advisory Committee on Fundamental Rights.

${ }^{7}$ On this see, $C A D$, Vol. VIII, 25 May 1949: 311, Appendix A

${ }^{8}$ Article 296 in the Draft Constitution originally contained: "In the all India and provincial services the claims of all minorities shall be kept in view in making appointments to these services consistently with the consideration of efficiency in administration".

9 CAD, Vol. X, 14 October 1949, p. 229

${ }^{10}$ CAD, Vol. X, 14 October 1949, p. 229

${ }^{11}$ See CAD, Vol. VII, 8 November 1948, p.323.

12 The First Backward Classes Commission (1953-55) held that "it would be not fair or just to list all Muslims as socially and educationally backward. Officially, Muslims do not recognize any caste. It must be said to the credit of Islam it did not compromise its position in the matter of untouchables. There are certain professions, however, that are regarded as inferior even by the Muslims. The sense of high and low has gradually permeated Muslim society and there are a number of communities amongst them that are suffering from social inferiority and consequent educational backwardness. We have recognized this deterioration that has overcome Muslim society today and added the names of such backward communities found 
among them in the list of Other Backward Classes". Government of India (1955): Report of the Backward Classes Commission (Vol.1), p. 27.

13 The mandate given to the committee broadly included: (a) to 'obtain relevant information from departments/agencies of Central \& State Governments and also conduct an intensive literature survey to identify published data, articles and research on relative social, economic and educational statistics of Muslims in India at the State, regional and district levels...', (b) to 'consolidate, collate and analyze the above information/literature to identify areas of intervention by Government to address relevant issues relating to the social, economic and educational status of the Muslim community'. Also see, Notification No. 850/3/C/3/05 - Pol. Government of India, New Delhi.

14 Report of National Commission on Religious and Linguistic Minorities (2007), Government of India, p. 153.

15 Although there is no constitutional limit to quantum of reservation, successive rulings of the Supreme Court beginning with Balaji Vs State of Mysore (1963) fixed a ceiling of 50 percent for all reservation taken together.

${ }^{16}$ For example, when the government of Andhra Pradesh announced a 5 per cent reservation for Muslims in the state in educational institutions and jobs in 2004, it raised political storms. The BJP described this reservation as 'anti-national' and announced to launch rigorous campaign against it. On July 18, Venkiah Naidu, a senior member of the BJP, told the reporters that 'the decision of to give 5 per cent of reservation to Muslims in education and jobs is dangerous, divisive and against national interest.'

17 For example, the Gujarat government refused to distribute scholarships initiated by Ministry of Minority Affairs to the minority students until the Gujarat High Court directed it to implement the scheme. Other BJP ruled states such as Chhattisgarh and Goa have also been reluctant to distribute scholarship to minority students,

18 See Ministry of Textiles, Government of India (also see, http://texmin.nic.in/policy/policy_scheme.htmhttp://texmin.nic.in)

19 Report of the Expert Group 'Equal Opportunity Commission: What, Why and How?' (2008), p. xvii. Government of India.

\section{References}

Ahmad, I. (ed.) (1978). Caste and Social Stratification among Muslims. New Delhi: Manohar

Ahmad, Z. (2013). Marginal Occupations and Modernizing Cities: Muslim

Butchers in Urban India. Economic and Political Weekly, 48(32), 121-30

Akhtar, S., \& N. Ahmad (2003). Reservation for Muslims: A Need of Hour, in S.N. Singh, (ed.) Muslims in India (pp. 37-66). New Delhi: Anmol Publications

Alam, M. S. (2009a). Backwards Logic. Indian Express, February 6. 
270 Alam - Affirmative Actions for Minorities in India

Alam, M. S. (2009b). Is Relative Size of Minority Linked to Underdevelopment?. Economic and Political Weekly, 44(48), 17-21. Retrieved from http://www.jstor.org/stable/25663829

Alam, M. S. (2010). Social Exclusion of Muslims in India and Deficient Debates about Affirmative Action. South Asia Research, 30(1), 43-65. doi: 10.1177/026272800903000103

Alam, M. S. (2014). Affirmative Action for Muslims: Arguments, Contentious and Alternatives. Studies in Indian Politics, 2(2), 215-229. doi: $10.1177 / 2321023014551877$

Ali, M (2013). Post- Sachar Reservation Politics', Economic and Political Weekly, 48(39), 13-15. Retrieved from http://www.cbgaindia.org/wpcontent/uploads/2016/05/Post-Sachar-Reservation-Politics-1.pdf Ali, Zaheer (2006). The Quota Trap. Economic and Political Weekly, 41(52), 5304-5306.

Ali, Anwar (2001). Maswat Ki Jung [in Urdu]. New Delhi: Vani Prakashan. Borooah, V.K. (2010). Social Exclusion and Job Reservation in India. Economic and Political Weekly, 45(52), 31-35. Retrieved from http://www.jstor.org/stable/27917960

Brest, P., \& Oshige, M. (1995) Affirmative Action for Whom? Stanford Law Review, 47(5), 855-900. Retrieved from

http://www.jstor.org/stable/1229177

Engineer, A. A. (2004). Reservation for Muslims. Economic and Political Weekly, 39 (36), $3984-85$.

Galanter, M. (1984). Competing Inequalities: Law and the Backward Classes in India. New Delhi: Oxford University Press

Galanter, M. (1978). Who Are the Other Backward Classes? An Introduction to a Constitutional Puzzle. Economic and Political Weekly, 13(43/44), 1812-1828. Retrieved from http://www.jstor.org/stable/4367065 Jamil, G. (2014). The Capitalist Logic of Spatial Segregation: A Study of Muslims in Delhi, Economic and Political Weekly, 49(3), 52-58.

Retrieved from http://www.epw.in/journal/2014/3/specialarticles/capitalist-logic-spatial-segregation.html

Jenkins, Laura D. (2003). Identity and Identification in India: Defining the Disadvantaged. London: Routledge Curzon

Harris-White, B. (2003). India Working, Cambridge: Cambridge University Press 
Hasan, Z. (2005). Reservation for Muslims, Seminar, No. 549. Retrieved from http://www.india-seminar.com/2005/549/549 zoya hassan.htm

Hasan, Z. (2009). Politics of Inclusion: Caste, Minorities and Affirmative Action. New Delhi: Oxford University Press

Karanath, D (2007). 'Caste among Indian Muslims' in A.H Ansari (Ed.), Basic Problems of OBC and Dalit Muslims, New Delhi: Serial Publications

Khan, J. A., \& Parvati, P. (2013). Government's Commitment towards Development of Muslims: A Post- Sachar Assessment of Uttar Pradesh and Haryana', in Z. Hasan \& M. Hasan (eds) India: Social Development Report, New Delhi: Oxford University Press

Krishnan, P. S. (2012). Reservation for Muslims in India: A Step for Inclusive Development. Economic and Political Weekly, 47(33), 60-65. Retrieved from http://www.epw.in/journal/2012/33/specialarticles/reservations-muslims-india.html

Kumar, D. (1992). Affirmative Action Debate in India. Asian Survey, 32(3), 290-302. Retrieved from http://www.jstor.org/stable/2644940

Khaitan, T. (2008). Transcending Reservation: A Paradigm Shift in the Debate of Equality. Economic and Political Weekly, 20(8), 8-12.

Retrieved from http://papers.ssrn.com/sol3/papers.cfm?abstract_id=1412503

Mondel, S.R (2003). Social Structure, OBC and Muslims, Economic and Political Weekly, 38 (46), 4892-97

Mullen, T. (1988). Affirmative Action. In S. Mclean \& N. Burrows (eds.) The Legal Relevance of Gender (pp. 244-66). Atlantic Highlands:

Humanities Press International.

Report of the National Commission on Religious and Linguistic Minorities (2007), Government of India

Report of the Backward Class Commission (1953), Manager, Government of India Press, Shimla

Report of the Backward Class Commission (1980), Government of India, Government Press, Delhi

Report of the Expert Group 'Equal Opportunity Commission: What, Why and How?', Government of India, New delhi

Sachar Committee Report (2006). Government of India, Cabinet Secretariat, New Delhi 
272 Alam - Affirmative Actions for Minorities in India

Shah, G. (1991). Social Backwardness and Politics of Reservation.

Economic and Political Weekly, 26(11/12), 601 - 610. Retrieved from http://www.jstor.org/stable/4397417

Shariff, Abusaleh \& Rakesh Basant (Eds.) (2010): Handbook of Indian

Muslims: Empirical and Policy Perspectives, New Delhi: Oxford University Press

Sowell, T. (2004). Affirmative Action Around the World. Yale: Yale University Press

Tejani, S. (2013). Between Inequality and Identity: The Indian Constituent Assembly and Religious Difference, 1946-50'. South Asia Research, 33(3), 205-221. doi: 10.1177/0262728013504675

Tierney, W. G. (1997). The Parameters of Affirmative Action: Equity and

Excellence in the Academy. Review of Educational Research, 67(2), 165-

96. doi: 10.3102/00346543067002165

Weiner, M. (1983). Political Consequences of Preferential Policies: A

Comparative Perspective. Comparative Politics, 16(1), 35-52. Retrieved from http://www.jstor.org/stable/421594

Weisskopf, Th. E. (2004). Affirmative Action in the United States and India: A Comparative Perspective. London: Routledge.

Wright, Th. P. Jr. (1997). A New Demand for Muslim Reservation in India. Asian Survey, 37(9), 852-8. Retrieved from http://www.jstor.org/stable/2645702

Md. Sanjeer Alam, Assistant profesor at Centre for the Study of Developing Societies, New Delhi.

Contact Address: Centre for the Study of Developing Societies (CSDS) 29, Rajpur Road, Delhi - 110054

Email : sanjeer@csds.in 


\section{HSE}

Historia Social y de la Educación

Social and Education History
Hipatia Press

www.hipatiapress.com

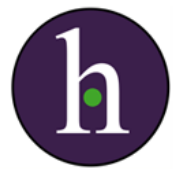

Instructions for authors, subscriptions and further details:

$\underline{\text { http://hse.hipatiapress.com }}$

Seeking new paradigms in aboriginal education research: methodological opportunities, challenges and aspirations

Marnee Shay ${ }^{1}$

1) Queensland University of Technology (Australia)

Date of publication: October 23rd, 2016

Edition period: October 2016 - February 2016

To cite this article: Shay, M. (2016). Seeking new paradigms in aboriginal education research: methodological opportunities, challenges and aspirations. Social and Education History 5(3), 273-296. doi:10.17583/hse.2016.2299

To link this article: http://dx.doi.org/10.17583/hse.2016.2299

PLEASE SCROLL DOWN FOR ARTICLE

The terms and conditions of use are related to the Open Journal System and to Creative Commons Attribution License (CC-BY). 


\title{
Seeking New Paradigms in Aboriginal Education Research: Methodological Opportunities, Challenges and Aspirations
}

\author{
Marnee Shay \\ Queensland University of Technology \\ (Australia)
}

\section{Abstract}

It is only relatively recently that Aboriginal peoples in Australia are represented in the academe, creating knowledges that speak for, and not of us. Internationally renowned Maori scholar, Professor Linda Tuhiwai Smith, was groundbreaking in her use of critical discourses needed for indigenous peoples globally to reclaim our knowledges and experiences through research. The emergence of Aboriginal and Torres Strait Islander scholars in Australia presents hope and opportunities for our communities to utilise the possibilities that ethical, Indigenous-driven research can have in interrogating complex and ongoing issues created by colonialism. Research that theorises Aboriginal epistemic and ontological research paradigms in Australia are still developing. Moreover, discipline-specific theoretical frameworks and methodologies are still emerging. This paper outlines my experience as an Aboriginal researcher in utilising Indigenist theoretical principles and methodological approaches. Using autobiographical reflections from my doctoral research, I present a range of practical implications that arise when the 'researched' shifts to the researcher in Western-dominated spaces such as schools. I will critically analyse the question, are Aboriginal researchers able to conduct research that is motivated by our agendas, ideas and aspirations in a discipline that perpetuates imperialism, racism and exclusion?

Keywords: indigenous, aboriginal, education research, access, gatekeeping 


\title{
La Búsqueda de Nuevos Paradigmas en la Investigación de la Educación
} Aborigen: Oportunidades Metodológicas, Desafíos y Aspiraciones

\author{
Marnee Shay \\ Queensland University of Technology \\ (Australia)
}

\section{Resumen}

Hace relativamente poco tiempo que los pueblos aborígenes de Australia han empezado a estar representados en el mundo académico, creando conocimientos que hablan por y no sobre nosotros. La profesora Linda Tuhiwai Smith, investigadora maorí internacionalmente reconocida, fue pionera en su uso de los discursos críticos necesarios para los pueblos indígenas en todo el mundo para reclamar nuestros conocimientos y experiencias a través de la investigación. La aparición de académicos aborígenes e isleños de Torres Strait en Australia aporta esperanza y oportunidades a nuestras comunidades para aprovechar las posibilidades que la investigación ética e impulsada por las propias personas indígenas puede aportar en el cuestionamiento de aspectos complejos y persistentes creados por el colonialismo. La investigación que teoriza los paradigmas epistemológicos y ontológicos de la investigación aborigen en Australia aún se están desarrollando. Además, los marcos teóricos y las metodologías específicas en la disciplina aún están emergiendo. Este artículo describe mi experiencia como investigadora aborigen en la utilización de los principios teóricos indigenistas y los enfoques metodológicos. A través del uso de reflexiones autobiográficas extraídas de mi investigación doctoral, presento una serie de implicaciones prácticas que surgen cuando el "investigado" pasa a ser el investigador en espacios dominados por occidentales como las escuelas. Analizaré críticamente la cuestión: los investigadores e investigadoras aborígenes, ¿somos capaces de llevar a cabo investigaciones motivadas por nuestros criterios, ideas y aspiraciones en una disciplina que perpetúa el imperialismo, el racismo y la exclusión?

Palabras clave: indígena, aborigen, investigación educativa, acceso, gatekeeping 
I am an early career Aboriginal researcher. My people are from Wagiman country in Daly River, Northern Territory through my Mother and Grandmother. Professionally, I have a youth work and teaching background. My teaching experience was predominantly in an education setting called 'Flexi Schools'. Flexi schools provide young people who have been disenfranchised from education the opportunity to reengage with learning (Shay, 2015). My research work has predominantly been in the Flexi Schooling context, with a particular focus on Indigenous engagement and experiences in this educational setting.

In this paper, I will use some of my autobiographical research reflections from my doctoral research, which explored the roles and experiences of Indigenous staff working in Flexi Schools. I took the position early on in conceptualising my project that I would choose theoretical and methodological approaches that connected to who I am and how I have experienced education as an Aboriginal person. I utilised Indigenous Standpoint Theory (Rigney, 2006) and Critical Race Theory (LadsonBillings, 1998) as the theoretical framework and utilised yarning as a methodology (Bessarab \& Ng'andu, 2010). Although it is beyond the scope of this paper to utilise my reflections of using yarning methodology, use of Indigenous methodologies such as yarning within educational research reveals the pervasiveness of Western paradigms even within 'Indigenous research'.

Due to the emerging nature of Indigenist research in Australia, it became clear at various times throughout undertaking this project that my reflections from doing this research may be just as much of a contribution as the data from the research itself. Bold (2012) discerns that some of the most insightful research is that which captures the whole story. In my doctoral study, I recognised the need to think and write as much about the process as the data itself. Aboriginal and Torres Strait Islander peoples are still establishing our own corpus of work that theorises knowledge production in a way that embodies our ways of knowing, being and doing (Martin, 2003). It is my aim to make a small contribution to the ongoing development of Indigenous scholarship.

In this paper, I will first outline my reflections about negotiating the space as an Aboriginal researcher in institutionalised education settings. I will discuss and provide an in-depth analysis of the issues that arise 
specifically for Indigenous researchers' access to research participants in institutionalised education settings. Furthermore, I will also raise some questions about existing Indigenous research ethical guidelines and the issues that they do not address for Indigenous researchers. The second topic to emerge from my reflection was gatekeeping in Indigenous education research and the specific implications for Indigenous researchers. I will use some examples of issues that have emerged from the broader gatekeeping literature to consider the explicit issues that potentially arise for Indigenous researchers who want to undertake Indigenous research in education settings.

\section{Access, Ethics and Indigenist Research}

Owing to historical practices in research that dehumanised, objectified and excluded Indigenous peoples from knowledge produced about us (Rigney, 2001), there is now a body of literature that emphasises ethical practices in conducting research that involves Indigenous peoples and communities. The increasing presence of Indigenous researchers presents opportunity for ongoing debate about conducting research with an Indigenous focus, from Indigenous perspectives in addition to non-Indigenous perspectives. Although there is a body of literature that speaks to Indigenous researchers (Foley, 2003; Martin, 2003; Moreton-Robinson, 2003; Nakata, 2007; Rigney, 2006), much of the literature speaks to and for non-Indigenous researchers undertaking Indigenous research.

There are two key documents that a researcher (Indigenous or nonIndigenous) must be familiar with if they are wanting to conduct research in Indigenous communities. The first ethical research guidelines for Indigenous research was developed by the National Health \& Medical Research Council (NHMRC) and published in 1991. This earlier version has now been replaced with 'Values and Ethics: Guidelines for Ethical Conduct in Aboriginal and Torres Strait Islander Health Research', published in 2003 (National Health \& Medical Research Council, 2003). The guidelines focus on research in Indigenous health contexts although they are often referred to by many researchers across disciplines. The NHMRC guidelines outline 
values and ethics needed for conduct of ethical Indigenous research including principles of reciprocity, respect, equality, responsibility, survival and protection and spirit and integrity (National Health \& Medical Research Council, 2003). The NHMRC emphasise relationships to counter the poor consultation that has occurred in the past with Indigenous participants of research. Moreover, the establishment of trust is also proposed as being central to shifting the power relationships that were the source of poor research relations between non-Indigenous researchers and Indigenous peoples.

The second key ethical framework was developed by the Australian Institute of Aboriginal and Torres Strait Islander Studies (AIATSIS). First published in 2002 and then updated and republished in 2012, the 'Guidelines of Ethical Research in Australian Indigenous Studies' is less health specific and provides researchers another set of recommendations for ethical research involving Indigenous peoples (Australian Institute of Aboriginal and Torres Strait Islander Studies, 2012). In the AIATSIS guidelines, fourteen principles are set out to frame how researchers should conduct ethical research with Indigenous peoples. The principles address issues such as rights and recognition of Indigenous peoples, consultation and informed consent, beneficence and outcomes serving the interests and needs of Indigenous peoples. AIATSIS cite human rights and self determination as the underlying principles for the development of these guidelines.

The guidelines and other literature that I engaged with when considering that my study will be Indigenous focused and only working with Indigenous participants, are written to a broad and what is assumed, mostly nonIndigenous audience. To me this places Aboriginal and Torres Strait Islander researchers in an ambiguous position. It can't be assumed that because we are Aboriginal or Torres Strait Islander that we will undertake research ethically. Notwithstanding, we are also circuitously bound into the very existence of the guidelines. Although we (Indigenous peoples) are now present and actively contributing to scholarship about us, the development of a body of literature that reflects the complexity of how we are currently positioned in the research space is still emerging.

I engaged with the AIATSIS and NHMRC guidelines when conceptualising this research and writing the ethics application for my project. The first gap I identified was that although I am Aboriginal and 
undertaking research which only includes Indigenous participants, this research was to take place in the specific context of schools. The institutionalised nature of education systems (both schools and universities) presents considerable issues in the practicalities of undertaking what I would name as Indigenist research in institutionalised contexts. Below is a reflection from my experience in navigating access to school sites for this research.

\section{Reflection}

Once I defined what flexi schools I want to work with in my study and why, I set about considering how I would work with participants. I had good existing relationships with some school communities because of my experience of teaching in multiple flexi schools. However, because I am now a researcher, how I approach Aboriginal and Torres Strait Islander staff to participate will need to be how the university dictates is an appropriate method for contacting potential participants. This was indeed a conundrum. My experience and knowledge as an Aboriginal person tells me very distinctively to ensure that I have consulted with mob on the ground, first and foremost. This did take place informally (well before I enrolled in a $P h D)$, which is how I knew that this research was something that Indigenous staff would see as valuable. However, I am now formally the researcher so bypassing formal hierarchies within the schools that I want to include in my study was not only a bad idea, it would be considered an unethical process.

Once I identified the schools, they were contacted via email as promised in my ethics approval. The way of negotiating forward differed because some flexi schools belonged to a network of more than one school and others were stand-alone independent schools where I had contacted the principal directly. I was only invited to meet face-to-face with one person who was in a high position systemically to make a decision about whether or not to grant access to Indigenous staff at their school sites. He was a white male manager with whom I had an existing relationship. We engaged in good critical conversation about what participation would involve, what my research questions were and discussion about my research design. This particular leader was emphatic of his support of the research. He made the 
decision in this meeting that he felt my research was so important that he would fund the cost of having Indigenous staff from multiple sites out from their schools for a day.

I drove away from that meeting feeling relieved. This man who held all of the authority to say yes or no to accessing Indigenous staff at multiple school sites (some of which have very high Indigenous enrolments) had not only said yes to supporting me to access participants, but also expressed that he valued the research I was about to embark on. In the car on the way home, I stopped to think - what if he had said no? What if he didn't know me, would that have impacted on his decision? If I was white, would he have still supported the very same study? That is great that he supports it, what if the school authorities below him don't support it?

As it turned out, the issues underlying some of the questions I asked myself on that drive home would continue to emerge as I contacted other schools that were stand-alone sites to recruit Indigenous participants. I had many schools who completely ignored my recruitment email. The ignoring of my email could only lead me to one of three conclusions. The first, that the principal discussed it with their Indigenous staff and they said no they weren't interested and the principal decided not to reply to communicate this. The second, that the principal didn't like what they read about the project and decided that they didn't want their Indigenous staff to be involved in such a study. The third, that the principal is too busy or saturated with research requests that they decided to ignore the email all together.

There were other replies. One was that there were not any Indigenous staff employed at the school currently. A legitimate reason not to be able to participate, I thought! The other was from a principal stating that they are not a flexi school. I took the time to reply and explain how I defined flexi schools (non-deficit) and that their school did offer education (with high Indigenous enrolments) that fits this definition but I did not get a reply to this email.

The principals and school leaders who worked directly under the man in the leadership position who had supported the study initially had mixed responses to my follow up communication. The majority were supportive in principle although the practical reality of having Indigenous staff away from their roles in schools surfaced as a very real barrier. Then there were others 
who were very proactive in passing information along and providing Indigenous staff the opportunity to participate in work time if they chose to.

\section{Institutionalised Education Settings and Indigenist Research}

The institutionalised nature of education presented very real implications and lessons for me as an Aboriginal researcher that I see as presently missing from Indigenous research literature. Indigenist research theories and methodologies are becoming a strong presence in the literature, although the translation of the theoretical principles to the practical reality of undertaking education research is not well documented. Ethical conduct of Indigenous research is emphasised through the guidelines discussed above by Australian Institute of Aboriginal and Torres Strait Islander Studies (2012) and the National Health \& Medical Research Council (2003). However, how are Aboriginal researchers able to conduct research that is motivated by our agendas, ideas and aspirations in a discipline and context that perpetuates imperialism, racism and exclusion?

My experience in navigating access to Indigenous participants in school sites guided me towards thinking about how Indigenous researchers negotiate institutionalised, western dominated spaces to undertake research that is proclaimed as being ethical in Indigenous research guidelines. Principles such as self-determination and consultation are nearing towards impossible when Indigenous researchers seek to undertake Indigenous research in education contexts and I would propose other institutionalised settings also. The regulating and governing of research agendas in education lays squarely with either bureaucrats in a large system or education leaders are in charge of individual school sites.

Schools have long been recognised in the literature as an institutionalised performative that functions far beyond that task of educating children (Berg, 2007; Jakobi, 2011; Ramirez \& Boli, 1987). Institutional theory "highlights cultural influences on decision making and formal structures" (Barley \& Tolbert, 1997, p. 93). Ramirez and Boli (1987) argue that the institutionalisation of education has resulted in the creation of mass schooling in almost every western European country. Moreover, Ramirez 
and Boli (1987) further discern that the purpose of mass schooling is "part of an endeavour to construct a unified national polity" (p. 3). Schools thus function as a mechanism to serve broader societal interests. Berg (2007) concludes that schools as institutions are then "the agency responsible for the reproduction of society, that is for instilling, e.g. social norms, cultural traditions, and the transmission of the knowledge and skills necessary to the individual and society" (p. 581). Analysis of the types of institutions and critique of how they discursively constitute the agendas of nation states are critical in education discourse.

The function of institutionalising schools is closely connected to the need for nation states to uphold national identities and values (Ramirez \& Boli, 1987). Nation states achieve their purpose of conformation through control, regulation and expected compliance by schools in upholding unity in 'shared' values and goals. This emphasis on national development in each individual school has resulted devaluing of the continuation of indigenous knowledges and principles, as well as diminishing of local and minority needs (Ramirez \& Boli, 1987).

Institutions such as schools reinforce dominant social norms, expectations and agendas. In an Australian context, the colonial project that originally saw Indigenous peoples rendered as sub-human through the declaration of 'terra nullius', continues to permeate through institutionalised, racial discourse in all institutions, including schools. Schools as institutions in Australia continue to uphold a national identity that ignores the brutality and dispossession of Indigenous peoples and constructs white Australians as the social norm. Some overt support of this statement is reflected in the data that demonstrates the under-representation of Indigenous principals, teachers, support staff or politicians who influence education policy (Australian Government, 2012, 2014; Lampert \& Burnett, 2012). Further evidence is in the western curriculum that has excluded teaching about the massacres and dispossession of Indigenous peoples in place of the 'Captain Cook' narrative of discovery of a great foreign land; the refusal to include teaching the many Aboriginal and Torres Strait Islander languages; the ongoing practices of deficit and stereotypical re-presentations by teachers of who Indigenous people are and the resistance to embedding of Indigenous perspectives and knowledges in the national curriculum (Ma Rhea, 2013; Phillips \& Lampert, 2012). 
Understanding how schools as institutions operate to serve the interests of the dominant culture is a critical aspect of the discussion about Indigenous researchers access to undertake Indigenous research in schools. For an Indigenous researcher to reach the position of being the researcher, one must: successfully navigate an institution as a child that is not functioning to support their interests or needs; access a different but similar institution to undertake tertiary studies (in an environment where we are even more critically under-represented); return to undertake research training where the likelihood of having someone teach you who is of the same cultural background is very low; and finally, construct a research project that affirms who you are culturally but also meets the needs of and is accepted by the institution. Once this is achieved, an Indigenous researcher who wants to undertake education research will then need to navigate the school institution again; this time, from the position of a researcher.

Shifting from the object of research to the researcher means a reconstruction of how we are socially positioned as Aboriginal peoples. In navigating schools as institutions, this means that Indigenous researchers will inevitably be met with similar issues as we were met with as students of the same institutions including being constructed as 'other'; inferior; subhuman and so forth. When Indigenous peoples become the researcher, it is not just the straight forward issues that arise with accessing school sites to undertake their research; there is a set of historical and social assumptions that we are structurally and individually met.

The notion of self determination, espoused by the two key ethical Indigenous research documents outlined earlier (AIATSIS and NHMRC) is therefore very unlikely to be possible in Indigenous education research. To consult with Indigenous people, create shared visions and collaborate with Indigenous peoples within education institutions is only possible if access is granted by the 'gatekeeper'. In school institutions, the gatekeeper will be the school leader (principal, lead teacher, head of campus) or higher up the bureaucracy within the system. In the Australian context, this person is unlikely to be an Aboriginal or Torres Strait Islander person, given how critically under-represented we are in educator and leadership roles (Australian Government, 2014). The role of gatekeeping within institutions 
will now be analysed. Such analysis is critical in bringing forward new ideas about ethics and new conversations for Indigenous researchers who want to undertake Indigenist research in education contexts.

\section{Gatekeeping and Indigenist Research in Education: Ethics or 'Protectionism'?}

Predictably, there is a body of literature on the concept of gatekeeping in research (Heath, Charles, Crow, \& Wiles, 2007; Murgatroyd, Karimi, Robinson, \& Rada, 2015; Sanghera \& Thapar-Björkert, 2008; Shoemaker \& Vos, 2009; Wanat, 2008). Gatekeeping of research has been written about across disciplines, with Wanat (2008) proposing that gaining access in a research context is unique to each study. The impartiality of the gatekeeping role has been noted in the literature for some time as being problematic (Shoemaker \& Vos, 2009). Murgatroyd et al. (2015) examined the role of gatekeeping in health research, identifying that gaps between practice and research will persevere with the "misuse of gatekeeping powers" (p. S163). Murgatroyd et al. (2015) further distinguish the misuse of gatekeeping roles as "Nimbyism"; a term which was used originally in the 1980's to describe residents who were in opposition of new developments in their neighbourhoods. Although residents agreed with the social outcomes that these developments might produce, they didn't want them in their neighbourhood. Thus, the term 'not in my backyard' was born. There are several layers to Nimbyism that impact on researchers that are defined by Murgatroyd et al. "Conditions of entry, defining the problem of study, access to data and respondents, funding and scope of analysis" (Murgatroyd et al., 2015, p. S163) are all influenced by Gatekeepers.

Heath et al. (2007) propose that gatekeepers can play an important ethical role, particularly for research involving children and other participants who are perceived as vulnerable. However, ethics in qualitative research include informed consent, which can only take place if potential participants have the opportunity to engage fully with the would-be researchers. Heath et al. (2007) further explain that in an institutionalised setting, most are "agestructured" (p. 405) consequently positing adults as authorities and decisionmakers. As institutionalised settings have a set of enforced conditions well 
outside the influence of potential participants or gatekeepers, Heath et al then question the ability to authentically gain informed consent if the participation is only decided upon by a gatekeeper. The issue that arises from this set of conditions is then about agency and decision making on behalf of potential participants. Status inequality, subordination and organisational constraints are all genuine issues that emerge when considering the ethics of gatekeeping and informed consent.

Sanghera and Thapar-Björkert (2008) conclude that the underlying dynamic that influences whether access is granted by a gatekeeper is the researcher-gatekeeper relationship, further outlining that "it is a relationship that is fraught with inconsistencies and instabilities" (p.544). Sanghera and Thapar-Björkert (2008) wrote of their research context, which was an inquiry of social capital in a complex, low socioeconomic community called Bradford in the United Kingdom. Ethnic diversity in the community is high and the researcher was wanting to research participants who may not have been of the same 'class' position, but likely of the same race categorisation. It was the position of the researcher that led to some rich insights about positionality (race, class, gender) and how it governs professional and social relationships. As the researcher was "British-born with Indian skin" (p.554), the researcher found himself fielding questions not only about his research, but about why he had brown skin and of Indian appearance but had a British accent. Accordingly, how gatekeepers constructed him as a person impacted on his relationships and interactions with him.

The unpredictability of how relationships are operationalised in gatekeeping/researcher interactions matters because it impacts on how knowledge is produced (Wanat, 2008). Wanat (2008) argues that high level gatekeepers tend to steer away from sensitive topics. Mediating access to participants is not only based on perceived benefits, it also based on perceived threats (organisational and individual). Wanat (2008) also raises the issue of translation of higher cooperation to lower level cooperation. Providing access through a systemic gatekeeper at a higher level does not always mean that the lower level will provide access to potential participants, particularly if the access isn't supported with resources or general support of the study. Wanat (2008) concluded that personal 
connections, at higher or lower levels in school research has the most impact on how researchers navigate gatekeeping.

Gatekeeping generally in Aboriginal and Torres Strait Islander communities is also not an unfamiliar concept. The Merriam Webster dictionary defines gatekeeping two ways. The first is defined as "a person who guards a gate". The second is defined as "a person who controls access" (Merriam-Webster, 2016). The metaphorical gate that can be applied when thinking about the concept of gatekeeping in Indigenous communities is the entry way to social, health, education, and economic equality that Indigenous peoples have fought for since invasion of Australia. Although who controls access to this equality is greater than just one person, the system that has blocked equality from being met over the past couple of centuries continues to prevent access to this equality. The person or persons who control the access are simply actors that are serving the interests of the very system that blocks access to equality, time and time again.

Historical policies such as protectionism are implicated in the gatekeeping that continues to pervade in many Indigenous communities around Australia. The protectionism rule that governed Indigenous peoples particularly from the early nineteenth century created a discourse that Indigenous peoples require protection or saving from ourselves and from others (Moran, 2005). What was initially a Government policy that was presented as preserving and safeguarding Indigenous peoples saw many thousands of Indigenous peoples removed from their traditional homelands and consequently separated from their families, language, and culture. Although the policy names (merging, absorption, assimilation) and ideology varied slightly over the subsequent decades up until the latter half of the nineteenth century, one consistent factor remained: the control and ruling of Indigenous lives by white missionaries, government officials and managers (Moran, 2005).

As outlined above, different scholars have written of their experiences of gatekeeping in varying discipline and context specific circumstances. All of this literature assists in critiquing how research and therefore knowledge production is mediated and for whose interest is the knowledge being created. In the context of Indigenous Australia, there are several key points in situ that I believe need to be raised for future Indigenous (and perhaps 
non-Indigenous researchers) in relation to gatekeeping Indigenist education research, by Indigenous researchers.

At the beginning of this section on gatekeeping, I cited the term Nimbyism, which referred to gatekeepers who might in essence support the social or moral value of the research, but 'not in their backyard' (Murgatroyd et al., 2015). In reference to Indigenist research conducted by Indigenous researchers, I believe this will continue to be an ongoing issue for some time to come. In the research context, we continue to see research that focuses on Indigenous learners as the problem; an abundance of research undertaken by non-Indigenous researchers researching the problematic 'other' and an ongoing obsession with comparative, scientific measurements of educational outcomes (Harrison, 2007).

Although many schools are now providing practices that are considered culturally inclusive, there remains a deficit discourse saturated with concepts of otherness that ensures Indigenous students are kept well below their nonIndigenous peers. The broader education policy space has seen some positive changes: the introduction of embedding Indigenous knowledges as a cross curriculum priority area (ACARA, 2015); the introduction of teacher standards that require teachers to know about Indigenous histories and have strategies to teach and support Indigenous students (AITSL, 2013) and increasing universities ensuring mandatory Indigenous education units within pre-service teacher education programs (Hart, Whatman, McLaughlin, \& Sharma-Brymer, 2012; Ma Rhea, 2013). Yet, these broader policy changes may not necessarily mean that Indigenist researchers who wish to undertake research in an education space on one of these topics will be granted access by a gatekeeper.

The school gatekeeper may be increasing their work in the area of Indigenous education. However, a study that might include critical observations or in depth analysis by the cultural 'other' may be perceived as useful, but 'not in their backyard'. In the context of this study, although I have no evidence beyond the correspondence between myself and gatekeepers, I believe this was an issue in some cases. I do not doubt the considerable limitations on school resources. However, a common issue that emerged at data collection at all sites was the ability to gain access to 
participants. Gatekeepers were quick to assure me how important the topic of Indigenous staff was, particularly due to the high Indigenous numbers of students and staff. Conversely, some gatekeepers only allowed access after ongoing persistence on my part or compromising on how I had planned to work with participants in collecting data to utilise the little time that was made available.

Murgatroyd et al. (2015) discussed the multiple aspects of control that gatekeepers have in research: "conditions of entry, defining the problem of the study, access to data and respondents, funding and scope of analysis" ( $p$. S165). The historically situated discourse in Indigenous education has always been socially and ideologically stipulated by white Australia. The conditions of entry in accessing education up until the latter end of the nineteenth century were clearly governed by racialised ideas that Indigenous peoples were intellectually inferior or "uneducable" (Price, 2012, p. 2). The conditions of entry into the space of knowledge production has not been different, with an emergence of Indigenous scholars writing of the challenges and their experiences of undertaking research within Western knowledge systems (Martin, 2012; Moreton-Robinson \& Walter, 2009; Nakata, 2007; Rigney, 2001).

In my study, my circumstances were that I had existing relationships with some school sites, which I believe impacted on how my conditions of entry were constructed in those cases. In others, the opportunity to discuss conditions of entry were blocked entirely without any prospect of negotiating or mediating with gatekeepers. The clear lack of neutrality in the role of gatekeepers in institutions such as schools with would-be Indigenist researchers such as myself, presents serious issues in being able to authentically consult and collaborate with Indigenous participants in school sites, as espoused by Indigenous research ethical guidelines and Indigenist theorists. With gatekeepers holding the authority to grant entry or place conditions upon entry, there is very little prospect for Indigenist researchers to define our own research problems and negotiate directly with participants about further defining the problem and the focus of the study. This is problematic because standpoint and how we perceive, observe and construct research problems, matters.

The abundance of research on Indigenous education has not resulted in any significant discoveries or improvements, and this research has been 
undertaken by mostly non-Indigenous researchers (Harrison, 2007). Counter stories are imperative, not just because it is essential to hear from those who hold the experiential knowledge; but also because experiential knowledge provides a different lens with which to construct and analyse the problem. Through the lived experiences of Indigenous peoples, the topic of race and racism often surfaces. Indigenous researchers often write of the importance of including the issue of race in relation to Indigenous scholarship (Carlson, 2011; Sarra, 2011). Critical race scholars also argue that specific examination of the role of race and racism, including schools and education systems, is vital in examining racial educational inequality that persists in many Western countries (Ladson-Billings, 1998; Ladson-Billings \& Tate, 2006; Solórzano \& Yosso, 2002; Zamudio, Russell, Rios, \& Bridgeman, 2011). The avoidance and conflated understanding of the topic of race and racism by educators is well documented (Aveling, 2002, 2007; Blackmore, 2010; Moreton-Robinson, Singh, Kolopenuk, Robinson, \& Walter, 2012). Bodkin-Andrews and Carlson (2014) evaluate that without insider or counter experiences outside of the realm of Western epistemologies framing inquiries on important topics such as race and racism, "it becomes apparent that the insidious effects of epistemological racism still plagues the Indigenous Australian educational research agenda" (p. 3).

Gatekeepers hold far more authority for Indigenous peoples than simply allowing or blocking research from being undertaken; they hold the authority to control how knowledge about us is produced and re-produced. Although the Australian Government promotes their resounding support for improving educational outcomes for Indigenous people, the lack of authority to control something as significant as knowledge production about us seems to be in complete contradiction to current education policy. As BodkinAndrews and Carlson (2014) point out, not only is it exclusionary by virtue of the dominance of Western knowledge systems, it also reproduces a different form of racism.

The role of ethics and gatekeeping are closely related. As mentioned earlier in this section, Heath et al. (2007) analysed the role of gatekeepers in gaining informed consent with children and vulnerable groups. Heath et al. critiqued positionality of potential participants, researchers and gatekeepers 
as problematic in undertaking the process of informed consent in research contexts. In their example, Heath et al. discussed the barrier of age structures within institutions in giving children the ability and agency to make decisions about their participation. Although the role of the gatekeeper is to protect children from being exploited thus bound with ethical research practices, it also has a paradoxical function of impeding a child's ability to be included in the decisions about them, that impinge on them.

Indigenous peoples in Australia are also categorised as a vulnerable group in research (Australian Institute of Aboriginal and Torres Strait Islander Studies, 2012; National Health \& Medical Research Council, 2003). The status of vulnerability is due to the extensive objectification, exploitation, exclusion and subjugation of us in research that have been conducted in the not-too-distant past (some would argue there are still examples of such studies) (Martin, 2012; Moreton-Robinson \& Walter, 2009; Rigney, 2006). While Heath et al. (2007) analyse age structures within institutions to consider how gatekeeping impacts on the rights and agency of children, intersections of age structures and race are important sites of causation to analyse when discussing ethics and Indigenist research.

Indigenous people have been racially constructed in Australia as inferior, other and less worthy since invasion (Moreton-Robinson, 2009). The social racialisation of Indigenous peoples and non-Indigenous peoples in Australia is then also connected with how gatekeepers undertake their ethical roles in 'protecting' us from further research that has not served our interests or accurately represented our lived experiences. Furthermore, protectionist discourse (that we need protecting from ourselves and others) must impinge on a gatekeeper's ability to reconcile the social construction of us needing protection with the 'vulnerable' Indigenous person now asking for access to their own group to undertake research. Social racialisation of White Australia implicitly tells a gatekeeper that they have more knowledge or authority to make decisions on behalf of the vulnerable Indigenous group that they are protecting. The invisible authority that is granted to make such decisions is constituted by virtue of how Whiteness discursively operates to keep Indigenous peoples subordinate thus maintaining the power and privileges that continue to benefit white people and systems (Blackmore, 2010; Moreton-Robinson, 2003). Recognition of this very real obstacle for Indigenous researchers who want to undertake research in institutionalised 
contexts such as schools is necessary to progress discussions about ethics and Indigenous research.

Wanat (2008) explains that gatekeepers in schools will often avoid topics that are sensitive. The topic of Indigenous peoples and affairs, in addition to racism could not only be categorised as being sensitive, but fraught with historically situated denial, untruths and assumptions. The difficulty in getting (non-Indigenous and White people in the main) to engage critically in Indigenous studies in education undergraduate programs has been written about by scholars such as Aveling (2002, 2006), Hart et al. (2012) and Phillips (2011). All of these authors stress the importance of compulsory Indigenous Studies in teacher education programs, yet acknowledge that students often enter the learning space with hostility, resistance and limited existing knowledge to draw on. The fear and resistance that exists in compulsory Indigenous education coursework is not limited to pre-service teachers.

Ma Rhea (2013) reported that there is widespread fear and concern amongst teachers nationwide in the recent policy changes that included mandatory teacher standards that require teachers to now know about Indigenous peoples, histories and cultures as well as to know how to effectively teach Indigenous students. Evidence is mounting that we currently have an education workforce who self-identify their deficiencies and lack of understanding about Indigenous peoples and issues. In relation to gatekeeping Indigenist research, the paramount question is, how are gatekeepers who likely have limited knowledge themselves about Indigenous peoples, cultures, communities and issues, able to make sound decisions about whether research (by Indigenous or non-Indigenous researchers) is appropriate and in the interests of their Indigenous students or staff? Moreover, researchers with specific experience and training in conducting Indigenist research are extremely limited. Leaving decisions to gatekeepers that have not engaged in any research training or have very limited understanding about the context of Indigenous research is not serving the interests of Indigenous peoples. 


\section{Conclusion}

In sum, I have used some of my reflections from my doctoral study to illustrate the need for expansion on current literature for Indigenous researchers who want to undertake education research. I briefly outlined two key documents, the NHMRC and AIATSIS ethics guidelines for conducting Indigenous research. My reflections and understanding of the ethics guidelines for undertaking Indigenous research revealed another gap in the literature whereby Indigenist researchers are using frameworks that are catering for a mostly non-Indigenous audience. In using my research reflections, I was able to critically analyse my experiences through examining literature on institutionalisation of education, gatekeeping and Indigenist research. Although I identified more problems than solutions, identifying where the issues are ended up being a critical aspect of this study in recognising the nexus between theory and practice in Indigenist research.

Indigenous researchers identify research problems and conceptualise research based on our diverse experiences as Indigenous peoples. I propose that Indigenous researchers need to continue to contribute to methodological and theoretical research literature through writing about our lived experiences as Indigenous researchers, providing insights for opportunities to overcome challenges and bring forth aspirations that exist in our communities. Finally, we need to create a body of scholarship that speaks to Indigenous researchers and provides practical solutions for the real issues that exist. The current Indigenous education focus on "Closing the Gap" must include the contribution that Indigenous researchers can make in solving complex issues created by colonialism.

\section{Notes}

${ }^{1}$ Terra Nullius - land belonging to nobody

\section{Acknoledgment}

This PhD is supervised by A/Prof Jo Lampert, A/Prof Grace Sarra and Aunty Denise Proud. Thanks and acknowledgement goes to my supervision team for their feedback on my research and their ongoing mentoring and guidance. 
292 Shay - Aboriginal Education Research

\section{References}

ACARA. (2015). Australia Curriculum. Retrieved from http://www.australiancurriculum.edu.au/

AITSL. (2013). Australian Professional Standards for Teachers. Retrieved from http://www.aitsl.edu.au/australian-professional-standards-forteachers/standards/list

Australian Government. (2012). Review of Higher Education Access and Outcomes for Aboriginal and Torres Strait Islander People Final Report. Retrieved from Retrieved from http://docs.education.gov.au/system/files/doc/other/heaccessandoutcomes foraboriginalandtorresstraitislanderfinalreport.pdf

Australian Government. (2014). Aboriginal and Torres Strait Islander Workforce Analysis More Aboriginal and Torres Strait Islander Teachers Initiative. Retrieved from http://matsiti.edu.au/wpcontent/uploads/2014/09/MATSITI-Data-Analysis-Report-2014.pdf

Australian Institute of Aboriginal and Torres Strait Islander Studies. (2012).

Guidelines for Ethical Research in Australian Indigenous Studies.

Retrieved from http://www.aiatsis.gov.au/_files/research/GERAIS.pdf Aveling, N. (2002). Student Teachers' Resistance to Exploring Racism:

Reflections on 'doing' border pedagogy. Asia-Pacific Journal of Teacher Education, 30(2), 119-130. doi:10.1080/13598660220135630

Aveling, N. (2006). 'Hacking at our very roots': rearticulating White racial identity within the context of teacher education. Race Ethnicity and Education, 9(3), 261-274. doi:10.1080/13613320600807576 Aveling, N. (2007). Anti-racism in Schools: A question of leadership?

Discourse: Studies in the Cultural Politics of Education, 28(1), 69-85. doi:10.1080/01596300601073630

Barley, S. R., \& Tolbert, P. S. (1997). Institutionalization and Structuration: Studying the Links between Action and Institution. Organization Studies, 18(1), 93-117. doi:10.1177/017084069701800106

Berg, G. (2007). From structural dilemmas to institutional imperatives: a descriptive theory of the school as an institution and of school organizations. Journal of Curriculum Studies, 39(5), 577-596. doi:10.1080/00220270600880994 
Bessarab, D., \& Ng'andu, B. (2010). Yarning about Yarning as a Legitimate Method in Indigenous Research. International Journal of Critical Indigenous Studies, 3(1), 37-50. Retrieved from

http://www.isrn.qut.edu.au/pdf/ijcis/v3n1_2010/Final_Bessarab_Bridget_ IJCIS.pdf

Blackmore, J. (2010). 'The Other Within': race/gender disruptions to the professional learning of white educational leaders. International Journal of Leadership in Education, 13(1), 45-61.

doi:10.1080/13603120903242931

Bodkin-Andrews, G., \& Carlson, B. (2014). The legacy of racism and Indigenous Australian identity within education. Race Ethnicity and Education, 1-24. doi:10.1080/13613324.2014.969224

Bold, C. (2012). Using Narrative in Research. Using Narrative in Research. London: SAGE Publications Ltd.

Carlson, B. (2011). The politics of identity: who counts as aboriginal today? (PhD), UNSW. Retrieved from

http://www.unsworks.unsw.edu.au/primo_library/libweb/action/dlDispla y.do?vid=UNSWORKS\&docId=unsworks_10196

Foley, D. (2003). Indigenous Epistemology and Indigenous Standpoint Theory. Social Alternatives, 22(1), 44-52.

Harrison, N. (2007). Where Do We Look Now?: The Future of Research in Indigenous Australian Education. Australian Journal of Indigenous Education, 36, 1-5.

Hart, V., Whatman, S., McLaughlin, J., \& Sharma-Brymer, V. (2012). Preservice teachers' pedagogical relationships and experiences of embedding Indigenous Australian knowledge in teaching practicum. Compare: A Journal of Comparative and International Education, 42(5), 703-723. doi:10.1080/03057925.2012.706480

Heath, S., Charles, V., Crow, G., \& Wiles, R. (2007). Informed Consent, Gatekeepers and Go-Betweens: Negotiating Consent in Child- and Youth-Orientated Institutions. British Educational Research Journal, 33(3), 403-417.

Jakobi, A. P. (2011). Political Parties and the Institutionalization of Education: A Comparative Analysis of Party Manifestos. Comparative Education Review, 55(2), 189-209. doi:10.1086/657931 
Ladson-Billings, G. (1998). Just what is critical race theory and what's it doing in a nice field like education? International Journal of Qualitative Studies in Education, 11(1), 7-24. doi:10.1080/095183998236863

Ladson Billings, G., \& Tate, W. (2006). Toward a critical race theory in education. In A. Dixson \& C. Rousseau (Eds.), Critical Race Theory in Education All god's children got a song (pp. 11-31). USA: Routledge. Lampert, J., \& Burnett, B. (2012). Retention and graduation of Aboriginal and Torres Strait Islander students in initial teacher education: a review of the literature. Paper presented at the Australian Teacher Education Association Annual Conference, Stamford Grand Hotel, Adelaide, SA. retrieved, November, 2014 from http://eprints.qut.edu.au/58067/

Ma Rhea, Z. (2013). Indigenous education and teacher professional development: the Australian professional standards for teacheres in Australia. Retrieved fom http://www.ieu.asn.au/media/71851/zane_ma_rhea1.pdf

Martin, K. (2003). Ways of knowing, being and doing: A theoretical framework and methods for indigenous and indigenist research. Journal of Australian Studies, 27(76), 203-214.

Martin, K. (2012). Please knock before you enter Aboriginal regulations of Outsiders and the implications for researchers. Teneriffe: Post Pressed.

Merriam-Webster. (2016). Online Dictionary. Retrieved from http://www.merriam-webster.com/dictionary/gatekeeper

Moran, A. (2005). White Australia, Settler Nationalism and Aboriginal Assimilation. Australian Journal of Politics \& History, 51(2), 168-193. doi:10.1111/j.1467-8497.2005.00369.x

Moreton-Robinson, A. (2003). Researching Whiteness: Some Reflections from an Indigenous Woman's Standpoint. Hecate, 29(2), 72-85.

Moreton-Robinson, A. (2009). imagining the good indigenous citizen: Race War and the Pathology of Patriarchal White Sovereignty. Cultural Studies Review, 15(2), 61-79.

Moreton-Robinson, A., Singh, D., Kolopenuk, J., Robinson, A., \& Walter, M. (2012). "Learning the Lessons? Pre-Service Teacher Preparation for Teaching Aboriginal and Torres Strait Islander Students" A Report prepared for the Division of Indigenous Education and Training Futures - 
Queensland Department of Education, Training and Employment, Indigenous Studies. Retrieved from http://www.aitsl.edu.au/docs/defaultsource/aitsl-research/insights/re00044_learning_the_lessons-_preservice_teacher_preparation_for_teaching_aboriginal_and_torres_strait_i slander_students_qut_oct_2012.pdf?sfvrsn=6

Moreton-Robinson, A., \& Walter, M. (2009). Indigenous methodologies in social research. Social research methods, 1-18.

Murgatroyd, P., Karimi, L., Robinson, P., \& Rada, J. (2015). On the use/misuse of health research gatekeeping powers in Australia: An underconsidered problem? Asia Pacific Journal of Health Management, 10(3), SI63-SI69.

Nakata, M. (2007). The Cultural Interface. Australian Journal of Indigenous Education, 36(Supplementary), 7-14. Retrieved from

http://search.informit.com.au.ezp01.library.qut.edu.au/documentSummar y; $\mathrm{dn}=903053619367156 ;$ res=IELIND

National Health \& Medical Research Council. (2003). Values and Ethics: Guidelines for Ethical Conduct in Aboriginal and Torres Strait Islander Health Research. Retrieved from

https://www.nhmrc.gov.au/_files_nhmrc/publications/attachments/e52.pd f

Phillips, J. (2011). Resisting contradictions : non-Indigenous pre-service teacher responses to critical Indigenous studies. Queensland University of Technology, Brisbane. Retrieved from http://eprints.qut.edu.au/46071/

Phillips, J., \& Lampert, J. (2012). Introductory Indigenous Studies in Education (2nd ed.). Frenchs Forest NSW: Pearson.

Price, K. (2012). A brief history of Aboriginal and Torres Strait Islander education in Australia Aboriginal and Torres Strait Islander education : an introduction for the teaching profession. New York: Cambridge University Press.

Ramirez, F. O., \& Boli, J. (1987). The Political Construction of Mass Schooling: European Origins and Worldwide Institutionalization. Sociology of Education, 60(1), 2-17.

Rigney, L. (2001). A first perspective of Indigenous Australian participation in science : framing Indigenous research towards Indigenous Australian intellectual sovereignty. Kaurna Higher Education Journal(7), 1-13. 
Rigney, L. (2006). Indigenist research and Aboriginal Australia. In N. Goduka \& J. Kunnie (Eds.), Indigenous people's wisdoms and power: Affirming our knowledges through narrative (pp. 32-50). London:

Ashgate Publishing.

Sanghera, G. S., \& Thapar-Björkert, S. (2008). Methodological dilemmas: gatekeepers and positionality in Bradford. Ethnic and Racial Studies, 31(3), 543-562. doi:10.1080/01419870701491952

Sarra, C. (2011). Strong and Smart - Towards a Pedagogy for Emancipation: Education for first peoples. Oxon: Routledge

Shay, M. (2015). The perceptions that shape us: strengthening Indigenous young people's cultural identity. In T. Ferfolja, Jones-Diaz, C. \& Ullman, J. (Ed.), Understanding sociological theory and pedagogical practices (pp. 93-105). Melbourne: Cambridge University Press.

Shoemaker, P. J., \& Vos, T. P. (2009). Gatekeeping theory (Vol. 1). GB: Routledge Ltd.

Solórzano, D. G., \& Yosso, T. J. (2002). Critical Race Methodology:

Counter-Storytelling as an Analytical Framework for Education

Research. Qualitative Inquiry, 8(1), 23-44.

doi:10.1177/107780040200800103

Wanat, C. L. (2008). Getting Past the Gatekeepers: Differences Between Access and Cooperation in Public School Research. Field Methods, 20(2), 191-208. doi:10.1177/1525822X07313811

Zamudio, M., Russell, C., Rios, F., \& Bridgeman, J. L. (2011). Critical Race Theory Matters: Education and Ideology Retrieved from http://QUT.eblib.com.au/patron/FullRecord.aspx?p=592906

Marnee Shay: lecturer in the School of Cultural and Professional Learning, Faculty of Education, Queensland University of Technology

Contact Address: ms.shay@qut.edu.au 


\section{HSE}

Historia Social y de la Educación

Social and Education History
Hipatia Press

www.hipatiapress.com

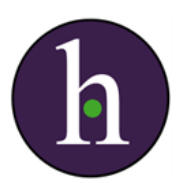

Instructions for authors, subscriptions and further details:

http://hse.hipatiapress.com

\section{A philosophical examination of social justice and child poverty}

Raquel Moreira ${ }^{1}$

1) Universidade Federal de São Carlos (Brasil).

Date of publication: October $23^{\text {rd }}, 2016$

Edition period: October 2016-February 2017

To cite this article: Moreira, R. (2016). A philosophical examination of social justice and child poverty [Review of the book]. Social and Education History 5(3), 297-299 doi:10.17583/hse.2016.2295

To link this article: http://dx.doi.org/10.17583/hse.2016.2295

\section{PLEASE SCROLL DOWN FOR ARTICLE}

The terms and conditions of use are related to the Open Journal System and to Creative Commons Attribution License (CC-BY). 
HSE - Social and Education History Vol.5 No. 3 October 2016 pp. 297-311

\section{Reviews (I)}

Schweiger, G., \& Graf, G. (2015). A philosophical examination of social justice and child poverty. Inglaterra: Palgrave Macmillan UK.

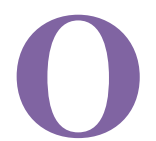

que significa ser pobre? Quais aspectos determinam a pobreza? Quais são os parâmetros para mensurar a pobreza? A pobreza pode determinar a vida de uma pessoa? Quem é responsável por ajudar a mudar as condições de vida dos pobres? A análise da pobreza e da justiça social é a mesma para crianças e adultos? Estas são algumas indagações e reflexões que Gottfried Schweiger e Gunter Graf abordam em "A philosophical examination of social justice and child poverty", para delinear a análise da pobreza infantil enquanto injustiça social.

Schweiger e Graf afirmam que a luta contra a pobreza infantil deve se respaldar em estudos multidisciplinares, combinando investigações críticas do ambiente econômico, social e político em que é produzida e reproduzida, com o modo que as instituições nacionais e internacionais agem para erradicá-la. Ressaltam que a filosofia pode contribuir, principalmente, a partir de aspectos normativos e éticos. Neste sentido, buscando ir além dos apontamentos da falta dos elementos básicos da vida, os autores delimitam o estudo da pobreza infantil no contexto dos países desenvolvidos, com foco numa avaliação moral do problema.

O livro conta com quatro capítulos. No primeiro, os autores traçam uma teoria da justiça social para crianças ancorada na abordagem das capacidades a partir de Amartya Sen e Martha Nussbaum. Destacando os conceitos de funcionamentos e de capacidades como essenciais para se fazer e ser autônomo, entendido como "bem-estar", eles afirmam que os 
funcionamentos são as atividades e os estados que compõem a vida de uma pessoa, garantindo à ela o acesso às capacidades para o "bem-estar", pautando a compreensão de justiça. Todavia, evidenciam a necessidade de um conceito de justiça específico às crianças, uma vez que elas não podem ser responsabilizadas por suas escolhas de vida tal como os adultos. Propõem um conceito normatizado pelos funcionamentos e não pelas capacidades, que deve operar na tensão do presente com o futuro, vislumbrando concomitantemente a criança enquanto criança e enquanto um futuro adulto. Com isto apresentam os conceitos de "bem-estar" e de "bemtornando-se" como categorias normativas, defendendo um limiar de funcionamentos que propiciem a igualdade de oportunidades ao acesso das capacidades necessárias ao "bem-estar".

No segundo capítulo, Schweiger e Graf examinam a pobreza infantil enquanto injustiça social. Respaldados pelos resultados das pesquisas empíricas, demonstram que a pobreza gera desvantagens corrosivas ao afetar negativamente mais de um funcionamento, comprometendo as capacidades para o "bem-estar" e o "bem-tornando-se". Junto a isso, evidenciam a saúde (mental e física), a educação e a inclusão social como funcionamentos férteis para outras funções e capacidades, utilizando-as como base de considerações normativas para mensurar a pobreza infantil.

Delimitado justiça social e pobreza infantil, os autores desenvolvem, no terceiro capítulo, um modelo de responsabilidades, tomando as contribuições de Iris Young e avançando numa proposta que parte dos aspectos de: causalidade, poder, privilégio e interesse. Ademais, distinguem oito grupos potenciais de agentes de justiça: a criança pobre, a família e cuidadores próximos, a comunidade de entorno e os ambientes sociais próximos, o Estado e suas instituições, a comunidade de cidadãos, a economia, a instituição internacional e a comunidade global. Mensurando, ao final, diferentes pesos para cada um, sem, ao mesmo tempo, desconsiderar a ampla responsabilidade de todos.

Compreendendo que o contexto de análise, países desenvolvidos, enviesa as argumentações e conceitos apresentados, os autores elencam, no último capítulo do livro, possíveis modificações para uma expansão da análise a nível mundial, a saber: delimitação dos limiares de funcionamentos a nível universal e redimensionamento das responsabilidades dos agentes de justiça. Encerram argumentando que muitas das defesas apresentadas podem ser mantidas e servir de base para avanços das questões de justiça e de pobreza 


\section{HSE - Social and Education History, 4(2) 299}

infantil a nível mundial, ressaltando, que sua erradicação efetiva só ocorrerá se tratada como um fenômeno global.

Salienta-se nesta obra o caráter propositivo normativo sobre pobreza infantil enquanto injustiça social, proporcionando a compreensão de que nos fazemos nas interações estabelecidas com outras pessoas e com o ambiente em que vivemos, repercutindo no que somos capazes de ser e fazer. Com isso, traz importantes contribuições às ações de erradicação da pobreza infantil e possibilita uma análise crítica acerca dos estudos do tema, oferecendo reflexões e destacando aspectos que precisam ser aprofundados em processo contínuo de investigação.

Raquel Moreira

Universidade Federal de São Carlos (Brasil)

profraquelmoreira@gmail.com 


\section{HSE}

Historia Social y de la Educación

Social and Education History
Hipatia Press

www.hipatiapress.com

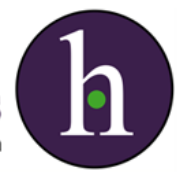

Instructions for authors, subscriptions and further details:

http://hse.hipatiapress.com

Culturally responsive leadership in Higher Education Promoting access, equity and improvement

Carlos Herrero $^{1}$

1) Universitat de Barcelona (Spain).

Date of publication: October 23rd 2016

Edition period: October 2016-February 2017

To cite this article: Herrero, C. (2016). Culturally Responsive Leadership in Higher Education. Promoting access, equity and Improvement [Review of the book]. Social and Education History 5(3), 300-303 doi:10.17583/hse.2016.2295

To link this article: http://dx.doi.org/10.17583/hse.2016.2260

\section{PLEASE SCROLL DOWN FOR ARTICLE}

The terms and conditions of use are related to the Open Journal System and to Creative Commons Attribution License (CC-BY). 
HSE - Social and Education History Vol.5 No. 3 October 2016 pp. 300-303

\section{Reviews (II)}

Santamaría, Lorry J. \& Santamaría, Andrés P. (Eds.). (2016). Culturally responsive leadership in higher education promoting access, equity and improvement. Routledge, New York.

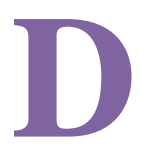

urante mi estancia en la Universidad de Auckland, Aotearoa - New Zeland, cayó en mis manos el libro Culturally Responsive Leadership in Higher Education. Promoting access, equity and Improvement. Un compendio de diferentes artículos escritos por veintiocho investigadores de diferentes áreas educativas y de diferentes procedencias (étnicas y culturales), que desarrollan y aplican en su día a día, el concepto de liderazgo crítico aplicado (Applied Critical Leadership) en diferentes campos de la educación superior y del mundo asociativo.

El presente libro está divido en cuatro grandes bloques de desarrollo, que van desde el hecho de romper con las desigualdades en la educación superior, la transformación de los líderes culturales en beneficio de los grupos desfavorecidos, el desarrollo de la equidad y la mejora del rendimiento académico, y la institucionalización de la responsabilidad cultural de los líderes en la implementación de la justicia social y la equidad. Cada uno de estos bloques recapitula en diferentes capítulos, las experiencias en primera persona de sus autoras, formando un tapiz interétnico de mujeres que forman una mezcla de colores, experiencias 
personales y trabajos que se tejen con sus relaciones profesionales y su contribución a la investigación a su vez.

Las diferentes secciones del libro se desarrollan en base a la investigación sobre las estructuras educativas en diferentes países (Estados Unidos de América, Australia y Aotearoa-Nueva Zelanda) y las desigualdades académicas, sociales y económicas que se producen con los grupos culturales y minorías étnicas de estos países (Afroamericanos, latinos, colectivo LGB, maorís y pacífica, entiéndase como los habitantes de las Islas del Pacífico que viven en Aotearoa-Nueva Zelanda).

Partiendo de este análisis de las desigualdades en la educación superior, urge la necesidad de llevar a cabo la transformación de las universidades a través de la creación de formas completarías de liderazgo, de dirección y administración, que promocionen la equidad y la justicia social, para la transformación del contexto social y el acceso de los grupos culturales y minorías étnicas que no han tenido las mismos oportunidades que la población blanca dominante. Para llevar a cabo la transformación este contexto centrado en las desigualdades raciales, culturales y de género, se argumenta que es necesario llevar a cabo una transformación del currículum educativo, en base a un concepto de pedagogía responsable, para el desarrollo de una filosofía de liderazgo, prácticas y políticas que creen ambientes para estudiantes y familias de diferentes bagajes étnicos $\mathrm{y}$ culturales.

La transformación social y cultural de estas instituciones parte de una transformación de los líderes de las mismas. Como se recoge en el libro, esta transformación se lleva a cabo a través de la participación de otros líderes de la comunidad, para aumentar la equidad del sistema. Con la transformación del liderazgo, a través de la participación de los otros líderes en ámbitos sociales y culturales, se deben identificar las desigualdades, reconociendo los errores, para acabar con las desigualdades y desarrollar un estado de reparación, reemplazo y de transformación, involucrando a los miembros de la comunidad. Esta transformación debe contemplar prácticas y estrategias 
que atiendan a las diferentes identidades (culturales y género) de sus participantes, así como potenciar la creación de sentido y de arraigo a las mismas.

Se destaca la importancia de la creación de un liderazgo crítico, que contribuya a cerrar la brecha educativa de los grupos desfavorecidos y que potencie prácticas y estrategias de liderazgo, que tome conciencia sobre las desigualdades creadas en educación, fomentando una pedagogía y un pensamiento crítico, en base al desarrollo de unas actividades centradas en la justicia social y de equidad, cubriendo las necesidades de los diferentes colectivos, para reducir el racismo y la exclusión social.

Para el desarrollo y la creación de personas líderes, se deben apoyar estrategias que apoyen a los grupos étnicos y culturales desfavorecidos el acceso a ocupar cargos en las universidades. Estas estrategias basadas en diferentes ámbitos de desarrollo; individual, profesional e institucional. A su vez se argumenta la necesidad de crear unos liderazgos críticos centrados en una mayor participación de las mujeres, sobretodo de aquellas tradicionalmente han sufrido una doble exclusión social, por el hecho de ser mujeres, y a su vez, de otras minorías o grupos étnicos socialmente excluidos. El hecho de dotar a las mujeres de espacios de decisión y participación, puede permitir romper con las relaciones desiguales de poder, reforzando el papel de estas mujeres como agentes de movilización y de acción colectiva. Estas mujeres parten de sus experiencias personales, su identidad y de su biografía personal, para llevar a cabo la transformación de las instituciones educativas y sociales. La creación de estas nuevas líderes críticas permite la democratización de las instituciones, así como se convierten en referentes para otras mujeres de su comunidad.

A lo largo del libro se plantea la superación de las barreras encontradas en los diferentes contextos académicos, que permita a estos grupos étnicos y culturales romper el techo de cristal que limita su acceso y potenciar su participación como agentes de capital social, para su transformación y fomentar una mayor democratización y justicia social de las instituciones académicas a través del liderazgo crítico aplicado. 


\section{HSE - Social and Education History, 4(2) 303}

Uno de los aspectos que creo que deben tener en cuentas estas mujeres en su camino hacia la creación de nuevas formas de liderazgo, es tener en cuenta a su vez la voz de las otras mujeres, que se basa en el desarrollo científico del feminismo dialógico, que tal y como se recoge en el libro de Lídia Puigvert, "las otras mujeres" (2001), aparte de desarrollar nuevos liderazgos, se deben crear espacios de participación para que se escuchen las voces de las otras mujeres, aquellas que hasta ahora no han tenido voz ni siquiera en el desarrollo del feminismo, trabajando unas líneas de diálogo igualitario, basado en la solidaridad, la creación de sentido y la igualdad de las diferencias, para llevar a cabo una profunda transformación de nuestras sociedades.

\section{Referencias}

Puigvert, L. (2001). Las otras mujeres. Barcelona: Hipatia Editorial.

Carlos Herrero Universitat de Barcelona (Spain) carlosherrero@ub.edu 